\title{
Exponential Error Suppression for Near-Term Quantum Devices
}

\author{
Bálint Koczor $\odot^{*}$ \\ Department of Materials, University of Oxford, Parks Road, Oxford OX1 3PH, United Kingdom
}

(Received 1 February 2021; revised 3 June 2021; accepted 30 July 2021; published 15 September 2021)

\begin{abstract}
Suppressing noise in physical systems is of fundamental importance. As quantum computers mature, quantum error correcting codes (QECs) will be adopted in order to suppress errors to any desired level. However in the noisy, intermediate-scale quantum (NISQ) era, the complexity and scale required to adopt even the smallest QEC is prohibitive: a single logical qubit needs to be encoded into many thousands of physical qubits. Here we show that, for the crucial case of estimating expectation values of observables (key to almost all NISQ algorithms) one can indeed achieve an effective exponential suppression. We take $n$ independently prepared circuit outputs to create a state whose symmetries prevent errors from contributing bias to the expected value. The approach is very well suited for current and near-term quantum devices as it is modular in the main computation and requires only a shallow circuit that bridges the $n$ copies immediately prior to measurement. Using no more than four circuit copies, we confirm error suppression below $10^{-6}$ for circuits consisting of several hundred noisy gates (2-qubit gate error $0.5 \%$ ) in numerical simulations validating our approach.
\end{abstract}

DOI: 10.1103/PhysRevX.11.031057

Subject Areas: Quantum Physics, Quantum Information

\section{INTRODUCTION}

The control of errors, also called noise, is fundamental to the successful exploitation of quantum computers. The powerful and general theory of quantum fault tolerance, exploiting quantum error correcting codes (QECs), provides a theoretical blueprint for controlling errors in the era when quantum devices are large scale [1-7]. Encoding qubits into collective states permits the suppression of the error rate on logical gates to an arbitrary small level at the cost of increasing the number of physical qubits. Below a threshold the error suppression is exponential in the hardware scaling. However, this powerful solution is prohibitive in the current era of noisy, intermediate-scale quantum (NISQ) devices for the following reasons [8]. (a) The qubit-count scale factor is at least 5 for the simplest codes that protect against comprehensive noise types $[9,10]$. (b) The extra circuit complexity that is needed in order to monitor the stabilizers, or equivalent measures of code integrity, is very considerable and will boost the effective error rate. (c) In order to achieve a universal set of quantum operations on code-protected logical qubits, highly nontrivial additional measures such as magic state

\footnotetext{
*balint.koczor@materials.ox.ac.uk
}

Published by the American Physical Society under the terms of the Creative Commons Attribution 4.0 International license. Further distribution of this work must maintain attribution to the author(s) and the published article's title, journal citation, and DOI. purification must be undertaken, greatly increasing the hardware scale.

Here we present an approach to controlling errors that achieves the key benefit of true QEC in the specific (but pivotal) case of estimating expected values of operators, and does so without the three key drawbacks of QEC mentioned above. The present idea requires an increased qubit count (by some integer factor that is at least two), and therefore it is more hardware expensive than many NISQ error mitigation schemes [11-21], but in return it provides exponential error suppression-which other NISQ solutions cannot. Therefore the approach might be seen as sitting between the established NISQ-era techniques and the full QEC domain, albeit nearer to the NISQ approaches. Moreover, the present approach is compatible with other NISQ mitigation techniques such as extrapolation, quasiprobability, or symmetry verification $[12,14-20]$; in fact, extrapolation is used in the present analysis to negate the impact of errors in the derangement process.

\section{A. Estimating expectation values}

Estimating expectation values on a quantum device is of central importance and most near-term applications do need to estimate such expectation values. Many variants of the so-called variational quantum eigensolver have been proposed for solving classically intractable problems, such as simulating quantum systems described by Hamiltonians $\mathcal{H}$ [11-14,16,22-41]. Expectation values of Hamiltonian operators are typically decomposed as $\left\langle\psi_{\text {id }}|\mathcal{H}| \psi_{\text {id }}\right\rangle=$ $\sum_{k} c_{k}\left\langle\psi_{\text {id }}\left|P_{k}\right| \psi_{\text {id }}\right\rangle$, where $P_{k}$ are tensor products of 
Pauli operators, and we collectively denote them as $\sigma \equiv P_{k}$ in the following. Various approaches have been proposed for estimating such expectation values $\left\langle\psi_{\text {id }}|\sigma| \psi_{\text {id }}\right\rangle$ using quantum computers [12,14,42-44]. However, without comprehensive error correction, errors during the state preparation will contribute a bias as $\left\langle\psi_{k}|\sigma| \psi_{k}\right\rangle$ into the result, where $\left|\psi_{k}\right\rangle$ are erroneous states, as shown in the next section. There exist numerous error mitigation techniques that potentially reduce the effect of such contributions without increasing the number $N$ of qubits, but at the cost of a significantly increased number of measurements and increased numbers of circuit variants [12,14-20]. Note that error mitigation techniques are also limited to correcting errors in measurements of observables as opposed to QECs.

Here we take a different route and introduce the error suppression by derangement (ESD) approach: we introduce a high degree of symmetry by preparing $n$ copies of the quantum state $|\psi\rangle$ and use derangement operators (generalized SWAP operations) to protect collective permutation symmetry. Most noise events that occur during the imperfect preparation of $|\psi\rangle$ break this permutation symmetry and they are effectively "filtered out" by the ESD. We outline a possible construction for such a measurement process in Fig. 1 and thoroughly analyze its properties while supporting our claims with rigorous mathematical proofs.

A crucial element of typical NISQ applications is the accurate estimation of expectation values of observables. The present approach allows one to exponentially suppress errors in such estimations and thus enables one to push the limits of a vast number of promising NISQ techniques. Let us name a few potential applications: variants of the

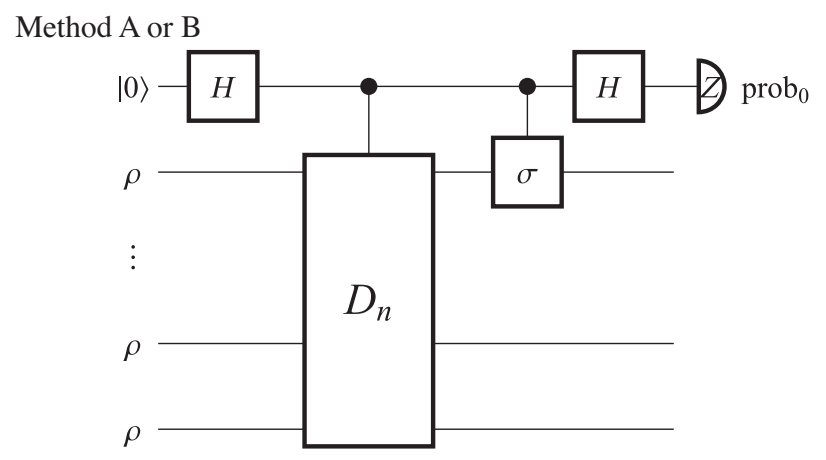

FIG. 1. A possible implementation of the ESD approach. Our derangement operator $D_{n}$ is a generalization of the SWAP operator and acts on $n$ (not necessarily identical) copies of the quantum state $\rho=\lambda|\psi\rangle\langle\psi|+(1-\lambda) \rho_{\mathrm{err}}$. In the above circuit $D_{n}$ permutes the $n$ registers and allows only permutation symmetric combinations, e.g., $|\psi\rangle^{\otimes n}$, to contribute to the expectation-value measurement process. The probability of measuring the ancilla qubit in the 0 state enables us to approximate the expectation value $\langle\psi|\sigma| \psi\rangle$ and errors are suppressed exponentially in $n$. This derangement operator can be implemented as a shallow circuit using a linear number $N(n-1)$ of primitive, controlled, 2-qubit SWAP gates. variational quantum eigensolver for, e.g., finding ground states of molecular Hamiltonians in quantum chemistry or spin model Hamiltonians in materials science; quantum approximate optimizations of graph problems; quantum machine learning and beyond. Please refer to the review articles [11-13] and references therein for more examples. Since the present approach is completely general and can be applied to the estimation of any observable (as discussed above), we present our results and proofs in complete generality without explicitly specifying or restricting the observable $\sigma$.

Our construction is certainly very well suited for NISQ hardware for the following reasons. First, the main computation is modular as the $n$ copies of the computational state are prepared completely independently. Second, the derangement circuit that "bridges" the $n$ copies immediately prior to measurements is sufficiently shallow (as it can be decomposed into a linear number of primitive gates) and therefore picks up significantly less noise than the statepreparation stage. Third, the derangement measurement is highly resilient to noise, since most error events that occur during the derangement process do not contribute to the result. Let us now introduce basic concepts and explain the main idea in detail.

\section{PRELIMINARIES}

\section{A. Noisy quantum states and entropies}

Near-term quantum devices aim to prepare computational quantum states $\left|\psi_{\text {id }}\right\rangle$ for, e.g., simulating other quantum systems or beyond. These quantum devices are, however, imperfect and can only prepare noisy, mixed quantum states which can be expressed generally via the spectral decomposition of a density matrix:

$$
\rho=\lambda|\psi\rangle\left\langle\psi\left|+(1-\lambda) \sum_{k=2}^{2^{N}} p_{k}\right| \psi_{k}\right\rangle\left\langle\psi_{k}\right| .
$$

Here $\lambda \leq 1$ and $\sum_{k} p_{k}=1$ is a probability distribution. It is important to recognize that the dominant eigenvector $|\psi\rangle$ above is not necessarily equivalent to the state that one would obtain from an ideal computation; even purely incoherent error models result in a small coherent mismatch in the dominant eigenvector. A comprehensive analysis of this coherent mismatch is presented in Ref. [45] and strong theoretical guarantees are provided that it can be exponentially smaller than the buildup of the erroneous contributions $\left|\psi_{k}\right\rangle$. In the following we thus focus on estimating expectation values in the dominant eigenvector, while advantages of this approach are discussed in the "Note added."

We further stress that in principle $\lambda$ can be arbitrarily small, e.g., $\lambda=10^{-6}$, as long as it is the dominant component and larger then any other eigenvalue as $\lambda>(1-\lambda) p_{k}$ for all $k$. Although, for extremely low $\lambda$ other factors such as 
the sampling cost may of course become prohibitive in practice, as we discuss in later text.

Furthermore, $p_{k}$ are probabilities of "erroneous" contributions $\left|\psi_{k}\right\rangle$, and we refer to these (orthonormal) states as erroneous eigenvectors in the following and we denote their probability vector as $\underline{p}$. To keep our discussion completely general, we do not restrict the probability distribution $\underline{p}$ at all, but we remark that Rényi entropies [46] as

$$
H_{n}(\underline{p}):=\frac{1}{1-n} \ln \left[\sum_{k=2}^{2^{N}} p_{k}^{n}\right]
$$

will have a crucial effect on the efficacy of the technique, and, indeed, for typical experimental quantum systems one can expect that $H_{n}(\underline{p})$ are large.

\section{B. Main idea}

As discussed above, most applications targeting early quantum devices aim to estimate expectation values $\left\langle\psi_{\text {id }}|\sigma| \psi_{\text {id }}\right\rangle$ in a quantum state $\left|\psi_{\text {id }}\right\rangle$ prepared by an ideal noiseless quantum device. Measuring expectation values in the dominant eigenvector $\langle\psi|\sigma| \psi\rangle$ from Eq. (1) would give in practical scenarios a very good approximation [45]; however, erroneous eigenvectors during state preparation contribute bias $\left\langle\psi_{k}|\sigma| \psi_{k}\right\rangle$ to the estimated expectation values. Here we aim to suppress these contributions via the following novel principle. Let us prepare $n$ copies of the state $\rho$ from Eq. (1). The most likely event during state preparation is that we obtain the dominant eigenvector of the state: with a probability $\lambda^{n}$ the resulting state (immediately after state preparation) is $|\psi, \psi, \ldots, \psi\rangle$. Measuring the expectation value on the first register gives the desired result $\langle\psi, \ldots, \psi, \psi \mid \sigma \psi, \psi, \ldots, \psi\rangle=\langle\psi|\sigma| \psi\rangle$.

In complete generality, under arbitrary noise models, the second most likely event is that one of the registers, for example, the first register, is found in the orthogonal erroneous eigenvector of the density matrix $\left|\psi_{k}\right\rangle$; a measurement then returns the error term $\left\langle\psi_{k}, \ldots, \psi, \psi \mid \sigma \psi_{k}, \psi, \ldots, \psi\right\rangle=$ $\left\langle\psi_{k}|\sigma| \psi_{k}\right\rangle$. However, if one instead measures the expectation value of the product $\sigma \mathrm{SWAP}_{1 n}$, where $\mathrm{SWAP}_{1 n}$ swaps the registers 1 and $n$, we then obtain

$$
\left\langle\psi_{k}, \ldots, \psi, \psi \mid \sigma \psi, \psi, \ldots, \psi_{k}\right\rangle=\left\langle\psi_{k}|\sigma| \psi\right\rangle\left\langle\psi_{k} \mid \psi\right\rangle=0 .
$$

Here the SWAP operator changed the ordering of the registers as $\left|\psi_{k}, \psi, \ldots, \psi\right\rangle \rightarrow\left|\psi, \psi, \ldots, \psi_{k}\right\rangle$ and the result is 0 due to the orthogonality of the eigenvectors of the density matrix. We can straightforwardly generalize this idea to the case where all registers are swapped, allowing only permutationsymmetric states to contribute to the measurement of expectation values. We refer to this permutation operation as "derangement." Let us emphasize that the above argument is completely general and holds for any noise model. While one can certainly realize the above measurement principle in various different ways, we propose one such circuit in Fig. 1. We rigorously prove properties of this particular construction in result 1 , result 2 , and result 3 , but we stress that the current proposal is not limited to the circuit in Fig. 1 (and even Fig. 1 leaves room for various different physical implementations which we discuss in later text).

\section{RESULTS}

\section{A. Exponential error suppression}

Let us now formally state the main result of the present work. In particular, the circuit in Fig. 1 can be thought of as a Hadamard-test technique [1] that measures the expectation value of the product $\sigma D_{n}$, where the derangement operator $D_{n}$ permutes the $n$ input registers; as we explain in a later section and discuss that it only requires a linear number of primitive gates to construct. We prove in Theorem 1 that only permutation-symmetric combinations can pass through the derangement measurement in Fig. 1, such as the dominant eigenvector $|\psi\rangle^{\otimes n}$ (which happens with a probability $\lambda^{n}$ ) or states in which the same errors occurred to all registers $\left|\psi_{k}\right\rangle^{\otimes n}$ [which happen with probabilities $(1-\lambda)^{n} p_{k}^{n}$ ]. Our general result in Theorem 1 determines the probability prob $_{0}$ of measuring the ancilla qubit in Fig. 1 in the 0 state as

$$
\begin{aligned}
2 \operatorname{prob}_{0}-1 & =\operatorname{Tr}\left[\rho^{n} \sigma\right] \\
& =\lambda^{n}\langle\psi|\sigma| \psi\rangle+(1-\lambda)^{n} \sum_{k=2}^{2^{N}} p_{k}^{n}\left\langle\psi_{k}|\sigma| \psi_{k}\right\rangle,
\end{aligned}
$$

where the erroneous contributions $\left\langle\psi_{k}|\sigma| \psi_{k}\right\rangle$ are exponentially suppressed as we increase $n$.

Dividing by $\lambda^{n}$ allows one to approximate the expectation value of a unitary observable $\sigma^{2}=\mathrm{Id}$, or otherwise the real part of the expected value of a unitary operator. We work out two explicit results in example 1 and example 2 that demonstrate how the above scheme allows us to exponentially suppress the noise as we increase the number $n$ of copies of $\rho$ and how its efficacy depends on properties of the probability distribution $p_{k}$. Let us now state approximation errors of methods A and B.

Result 1.-Let us prepare $n$ identical copies of the experimental quantum state $\rho$ from Eq. (1) and apply the derangement measurement from Fig. 1. Both methods A and $\mathrm{B}$ approximate the expectation value $\langle\psi|\sigma| \psi\rangle$ by estimating prob $_{0}$ on the ancilla qubit. Method B only estimates prob $_{0}$ and assumes explicit knowledge of the dominant eigenvalue $\lambda$. In method $\mathrm{A}$ we additionally estimate $\operatorname{prob}_{0}^{\prime}$ by repeating the procedure but omitting the controlled- $\sigma$ gate in Fig. 1. We denote their approximation errors as $\mathcal{E}_{A}$ and $\mathcal{E}_{B}$, respectively,

$$
\text { Method A: } \frac{2 \operatorname{prob}_{0}-1}{2 \operatorname{prob}_{0}^{\prime}-1}=\langle\psi|\sigma| \psi\rangle+\mathcal{E}_{A},
$$




$$
\text { Method B : } \quad \frac{2 \operatorname{prob}_{0}-1}{\lambda^{n}}=\langle\psi|\sigma| \psi\rangle+\mathcal{E}_{B},
$$

and these approximation errors generally decay exponentially with the number $n$ of copies via the sequence $Q_{n}$,

$$
\left|\mathcal{E}_{A}\right| \leq \frac{2 Q_{n}}{1+Q_{n}} \quad \text { and } \quad\left|\mathcal{E}_{B}\right| \leq Q_{n}
$$

which is bounded $Q_{n} \leq$ const $\times Q^{n}$ via the suppression factor $Q<1$ as established in Theorem 2 and in Lemma 1.

Note that these error bounds naturally extend to observables $\mathcal{H}$ of unit norm that are linear combinations of Pauli strings. We show in Lemma 1 that the errors also decay exponentially with the Rényi entropy $H_{n}(\underline{p})$ of the error probability distribution from Eq. (1) via $Q_{n}=$ $\left(\lambda^{-1}-1\right)^{n} \exp \left[(1-n) H_{n}(\underline{p})\right]$. Even without knowing or having a good guess of the Rényi entropy of the error probabilities, we can state a general upper bound that only depends on the two largest eigenvalues of the state as $Q_{n} \leq\left(\lambda^{-1}-1\right)^{n}\left(p_{\max }\right)^{n-1}$, where $p_{\max }$ is the largest of the error probabilities $p_{k}$ in Eq. (1). Note that these quantities, and thus the upper bounds, may be estimated experimentally [47-53].

\section{B. Numerical simulations}

Let us now numerically verify the above bounds in a practical setting: We consider a 12-qubit quantum state that is produced by a noisy, parametrized quantum circuit typically used in variational quantum algorithms-our circuit consists of 10 alternating layers and overall 372 quantum gates. Refer to Appendix F for more details. Each 2-qubit gate undergoes 2-qubit depolarizing noise with $0.5 \%$ probability and each single-qubit gate undergoes depolarizing noise with $0.05 \%$ probability. The resulting state has a dominant eigenvalue $\lambda \approx 0.51$ and it has a high entropy, full-rank error probability distribution via the Rényi entropies that monotonically decrease with $n$ as $H_{2}(\underline{p})=4.69, H_{3}(\underline{p})=4.38, H_{4}(\underline{p})=4.23$, and $H_{\infty}(p)=3.63$. Refer to Appendix $\mathrm{F}$ for more details.

We remind the reader that despite the purely incoherent error model, the dominant eigenvector $|\psi\rangle$ of $\rho$ is slightly different than what one would obtain from a completely error-free computation and in Fig. 2 we compute errors using the dominant eigenvector; refer to Appendix F for more details.

In Fig. 2 (left) we plot our error suppression upper bounds from result 1; i.e., solid lines represent the error bounds computed from the Rényi entropy of the quantum state's error-probability distribution and dashed lines represent the general upper bound $Q_{n} \leq\left(\lambda^{-1}-1\right)^{n}\left(p_{\max }\right)^{n-1}$, where the largest error probability is $p_{\max }=0.026$ and the suppression factor is $Q=0.026$. Red and blue colors correspond to method A and method B, respectively. We have generated 500 Pauli strings as observables randomly and computed the errors in estimating their expectation values (there are overall $4^{12}=1.68 \times 10^{7}$ Pauli strings, and we randomly select 500). These samples (see horizontal lines in Fig. 2) are significantly below our upper bounds and seem to decrease in a similar exponential order as our bounds (i.e., slope is similar in the logarithmic plot).

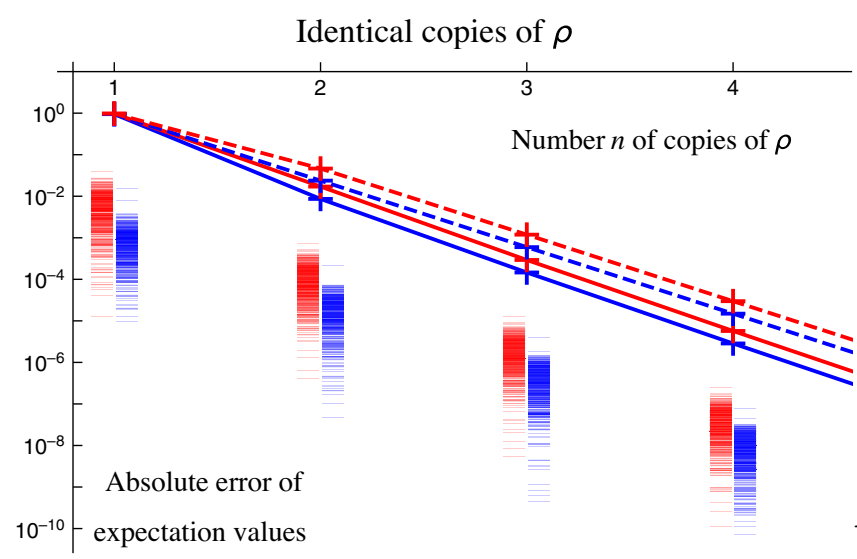

Red: Method A

Upper bounds with Rényi entropy

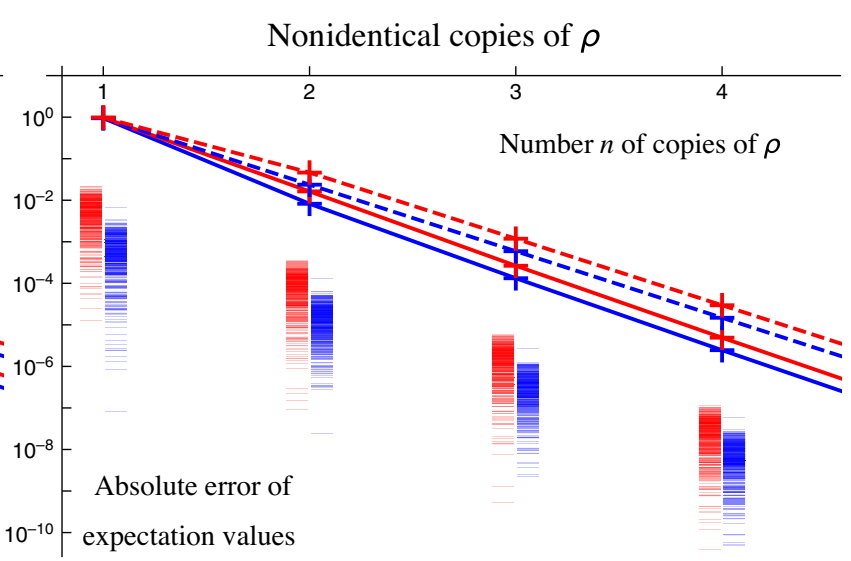

Blue: Method B

\section{--_-_.-General upper bounds}

FIG. 2. Simulation of a 12-qubit state with 372 noisy quantum gates. Errors in estimating the expectation value in the dominant eigenvector $\langle\psi|\sigma| \psi\rangle$ decay exponentially with the number of copies $n$ of the quantum state $\rho$. Dashed lines: our general upper bounds on the errors from result 1 and result 2 only require the knowledge of the dominant eigenvalue $\lambda$ and the largest error probability $p_{\text {max }}$ from Eq. (1). Solid lines: our upper bounds based on the Rényi entropy of the quantum states. Red and blue bars: approximation errors obtained with 500 randomly selected Pauli strings (observables $\sigma$ ) are significantly below the upper bounds. (left) All copies of $\rho$ are perfectly identical and (right) all copies of $\rho$ are significantly different (but they commute as this case can be simulated efficiently) and their trace distance is 0.01 due to their different eigenvalue distributions. 
Method B slightly outperforms method A (slightly smaller errors as blue is slightly below red), but it requires an exact (or very precise) knowledge of the dominant eigenvalue $\lambda$. Nevertheless, this eigenvalue could be determined precisely by existing approaches in special cases, e.g., as in Ref. [54].

\section{Effect of nonidentical states}

We now turn to the question of how the efficacy of our error suppression scheme is affected when the $n$ copies of the state $\rho$ are not identical.

Result 2.-We assume that all copies of the quantum state are arbitrarily different via $\rho_{1} \neq \rho_{2} \neq \cdots \rho_{n}$ except that their dominant eigenvector is $|\psi\rangle$. Our scheme via Lemma 3 still provides exponentially decreasing approximation errors when the dominant eigenvalue of the worst quality copy is $\lambda_{\min }>1 / 2$ via

Method A: $\frac{2 \operatorname{prob}_{0}-1}{2 \operatorname{prob}_{0}^{\prime}-1}=\langle\psi|\sigma| \psi\rangle+\mathcal{O}\left(\left[\lambda_{\text {min }}^{-1}-1\right]^{n}\right)$,

Method B : $\frac{2 \operatorname{prob}_{0}-1}{\prod_{\mu=1}^{n} \lambda_{\mu}}=\langle\psi|\sigma| \psi\rangle+\mathcal{O}\left(\left[\lambda_{\min }^{-1}-1\right]^{n}\right)$.

In the special case when all copies of the quantum state commute (same eigenvectors but different eigenvalues), one can expect very similar approximation errors to result 1 via an effective sequence $Q_{n}^{\text {eff }}$.

We can efficiently simulate the case when all copies of the quantum state commute. We disturbed every copy of the density matrix such that their trace distance is $\left\|\rho_{k}-\rho_{l}\right\| \approx$ $10^{-2}$ for all $k \neq l$. Note that the approximation errors in Fig. 2 (right) are very similar to Fig. 2 (left) and they are approximately upper bounded by the same upper bounds from result 1 (as expected from Lemma 3).

\section{Complexity analysis}

Let us now analyze resource requirements of our ESD approach. In particular, one needs to prepare a suitable number $n$ of copies of $\rho$ in order to suppress its errors below a threshold level, which we refer to as precision and denote as $\mathcal{E}$. The overall number of qubits required is then $n N+1$, where $N$ is the number of qubits in the computational state $\rho$. Furthermore, one needs to repeat measurements many times to sufficiently reduce the effect of so-called shot noise; i.e., we estimate the probability only from a finite number of repetitions [55]. We denote the number of repetitions as $N_{s}$. Let us now summarize our general results from Lemma 2.

Result 3.-In order to reach a precision $\mathcal{E}$ in determining the expectation value $\langle\psi|\sigma| \psi\rangle$, one requires a logarithmic number $n=\mathcal{O}\left(\ln \mathcal{E}^{-1} / \ln Q^{-1}\right)$ of copies of the quantum state $\rho$ (up to rounding). Here $Q<1$ is the suppression factor from result 1 that depends on Rényi entropies.
The number $N_{s}$ of measurements required to suppress shot noise below the threshold $\mathcal{E}$ grows polynomially as

$$
\begin{array}{ll}
\text { Method A : } & N_{s}=\mathcal{O}\left[\mathcal{E}^{-2(1+2 f)}\right], \\
\text { Method B : } & N_{s}=\mathcal{O}\left[\mathcal{E}^{-2(1+f)}\right],
\end{array}
$$

where $f=\ln \left[\lambda^{-1} / \ln Q^{-1}\right]$ increases the polynomial order compared to the standard shot-noise limit $\mathcal{O}\left(\mathcal{E}^{-2}\right)$ and we have derived a general upper bound on $f$ in Lemma 2 .

Dividing by the exponentially attenuated factor $\lambda^{n}$ in both methods $\mathrm{A}$ and $\mathrm{B}$ in result 1 requires an increasingly large number of measurements to sufficiently suppress shot noise. Methods $\mathrm{A}$ and $\mathrm{B}$ are therefore less efficient than permitted by the standard shot-noise limit $N_{s}=\mathcal{O}\left(\mathcal{E}^{-2}\right)$. For example, in the extreme, but still valid, case of $\lambda=$ $10^{-6}$ and $Q=1 / 2$, we obtain $f=19.9$ which increases the sampling costs prohibitively in practice. Nevertheless, the polynomial order of $\mathcal{O}\left(\mathcal{E}^{-1}\right)$ is only logarithmically increased via $f$ and its effect might be negligible in practically relevant scenarios. For example, in our simulations in Fig. 2 we obtain $f=0.18$ using our expression $Q=\left(\lambda^{-1}-1\right) p_{\max }$ in Lemma 1 . Indeed, we recover the standard shot-noise limit $\mathcal{O}\left(\mathcal{E}^{-2}\right)$ for very good quality states $\lambda \approx 1$ or for very high entropy probabilities.

In summary, the complexity of our ESD approach only depends on the largest eigenvalue $\lambda$ of the state and on the suppression factor $Q$ from result 1 -which is determined by the Rényi entropy of the error probabilities. As expected, the number $N_{s}$ of samples grows polynomially with the target precision $\mathcal{E}^{-1}$ and the system size (via $n$ ) grows logarithmically with $\mathcal{E}^{-1}$. Let us remark that in case of certain applications a global prefactor in observable expectation values does not matter-such as in case of variational quantum eigensolver (VQE) optimizationsand one can use method B but omitting the division by $\lambda^{n}$. Using method B significantly reduces the measurement costs and reduces errors from result 1 when compared to method A.

\section{E. Derangements of quantum registers}

Let us now discuss how to implement derangement circuits using a linearly growing number (in $n$ and $N$ ) elementary gate operations. In particular, our ESD circuit in Fig. 1 uses a generalization of the SWAP operator that permutes subspaces of quantum registers. Recall that in general there exist $n$ ! permutations of a set of $n$ ordered elements. Derangements are a subset of the collection of all permutations: they permute the $n$ elements such that no element remains in place [56,57]. We define $D_{n}$ in Definition 1 as unitary representations such that they permute subspaces of $n$ quantum registers. For example, for $n=2$ our $D_{2}$ reduces to the usual SWAP operator as

$$
D_{2}\left|\psi_{1}, \psi_{2}\right\rangle=\operatorname{SWAP}_{12}\left|\psi_{1}, \psi_{2}\right\rangle=\left|\psi_{2}, \psi_{1}\right\rangle .
$$


Note that here $\mathrm{SWAP}_{12}$ swaps the two registers, but it decomposes into $N$ elementary SWAP operations between pairs of qubits within the registers. For $n=3$ we have two distinct constructions for possible $D_{3}$ derangement operators as

$$
\begin{aligned}
& \operatorname{SWAP}_{13} \operatorname{SWAP}_{12}\left|\psi_{1}, \psi_{2}, \psi_{3}\right\rangle=\left|\psi_{3}, \psi_{1}, \psi_{2}\right\rangle, \\
& \operatorname{SWAP}_{23} \operatorname{SWAP}_{12}\left|\psi_{1}, \psi_{2}, \psi_{3}\right\rangle=\left|\psi_{2}, \psi_{3}, \psi_{1}\right\rangle .
\end{aligned}
$$

For $n=4$ one has 6 possibilities while in general there are $(n-1)$ ! possibilities for constructing distinct derangement operators - but choosing any one of these constructions is sufficient for our scheme to work. Indeed, one could construct derangements $D_{n}$ straightforwardly as cyclic shifts [47], but the large number of possibilities might offer more preferable constructions that take into account, e.g., hardware constraints such as connectivity. Refer to Ref. [58] for illustrations of the corresponding circuits. Furthermore, we discuss in Appendix E 2 that the large number of symmetries in the derangement circuit can be exploited in order to, e.g., reduce errors that happen during the controlled-sWAP operations.

Regarding gate complexity, derangement operators can be implemented efficiently in general using $N(n-1)$ elementary controlled 2-qubit SWAP gates, where $N$ is the number of qubits in the register $|\psi\rangle$ and $n$ is the number of copies of $|\psi\rangle$. These minimal SWAP circuits (which optimally implement derangement operators) can be constructed by mapping the corresponding permutations to graph trees [59]; refer to Definition 1.

It is important to recognize that while the number of elementary controlled-swAP gates grows as $\mathcal{O}(N)$, preparing the quantum state $|\psi\rangle$ generally requires $\mathcal{O}[a(N) N]$ gates, where $a(N)$ is the depth of the computation. It is generally expected that for practical problems one needs to go beyond constant-depth circuits such that the number of gates in the main computation grows faster than $\mathcal{O}(N)$ [60-64]. Thus the gate count of the derangement circuit can be expected to be of diminishing relative significance when scaling up computations. Even if the controlled-SWAP operator is not a hardware-native gate, one needs at most 6 native entangling gates to implement the elementary controlled-SWAP operator; see Table I in Appendix E. We demonstrate this below on a practical example assuming a hardware-native gate set and also briefly discuss connectivity constraints.

\section{NOISE ROBUSTNESS AND LIMITATIONS}

\section{A. Mitigating experimental imperfections}

So far we have assumed that the derangement operator in Fig. 1 is perfect. Indeed, gates involved here are expected to be noisy in a realistic scenario which ultimately limits the precision of our approach and increases its complexity.
We show in example 3 quite generally that the derangement operator is highly resilient to experimental imperfections and protects permutation symmetry even under experimental noise. This is nicely illustrated in our simulated noisy circuit: the unmitigated errors in determining prob $_{0}$ in Fig. 3 are quite low and are below $10^{-2}$ for all 50 randomly selected states. The simulated circuit consist of 13 qubits, i.e., 3 copies of a 4-qubit state, and elementary controlled-SWAP gates undergo 3-qubit depolarizations with a probability $3 \times 10^{-3}$. Refer to Appendix $\mathrm{F}$ for more details.

Most importantly, we show in example 3 quite generally that most errors that occur during the derangement measurement will only trivially affect the final result by (almost) linearly attenuating the output probability prob $_{0}$ which can in principle be corrected by an extrapolation. We use extrapolation techniques [12,14-17,65] which typically estimate $\operatorname{prob}_{0}(\epsilon)$ at different values of $\epsilon$ and extrapolate, e.g., linearly, to zero noise $\epsilon=0$. Because of the high degree of noise resilience of the derangement operator, the measurement probabilities $\operatorname{prob}_{0}(\epsilon)$ are closely approximated by a linear function in $\epsilon$, and Fig. 3 illustrates that indeed a linear extrapolation surprisingly well approximates the ideal probability with errors less than $10^{-4}$.

Here we aim to suppress errors arbitrarily by accounting for the slight nonlinearity of the function $\operatorname{prob}_{0}(\epsilon)$. We prove in Theorem 3 quite generally that expectation

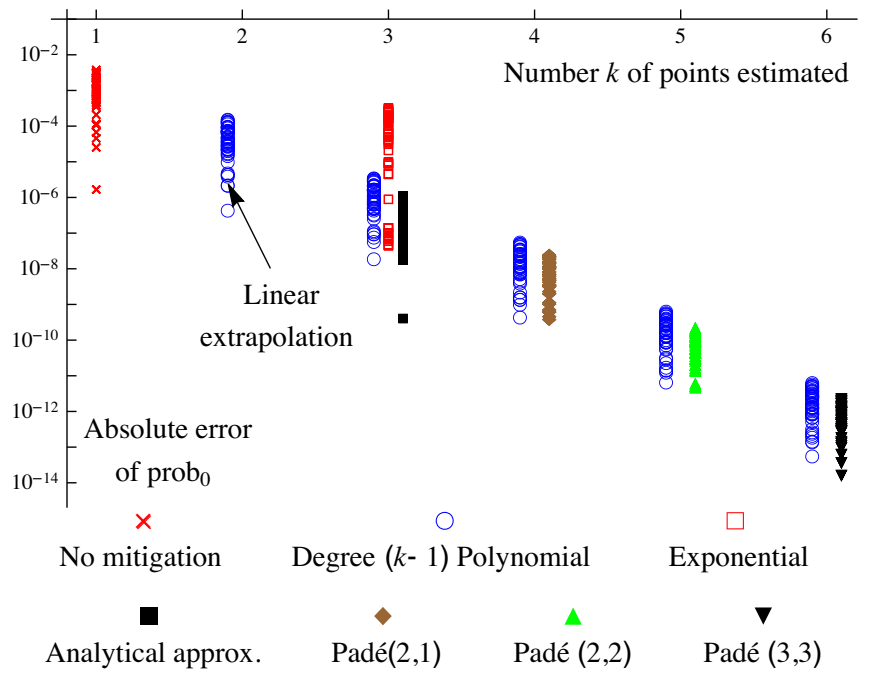

FIG. 3. Mitigating errors in the derangement operator. Extrapolation errors using various different fitting techniques versus the number of fitting points for 50 randomly selected Ansatz states. Elementary gates in the derangement operator in Fig. 1 have an error rate $\epsilon=10^{-3}$ and an experimentalist can increase this error in $k=2,3,4, \ldots$ steps up to $\epsilon=10^{-2}$. The probability prob $_{0}$ from Fig. 1 at $\epsilon=0$ is estimated by extrapolating to $\epsilon=0$. The derangement measurement is highly resilient to imperfections (see text) and $\operatorname{prob}_{0}(\epsilon)$ is almost linear in $\epsilon$. Increasing the degree of the fitting polynomial (blue circles) reduces the extrapolation error exponentially. 
values are exactly described by degree- $\nu$ polynomials as $\operatorname{prob}_{0}(\epsilon)=\sum_{k=0}^{\nu} c_{k} \epsilon^{k}$ and $\nu$ is the number of noisy gates. It follows that one can in principle determine the ideal probability by determining $\operatorname{prob}_{0}(\epsilon)$ at $\nu+1$ different values of $\epsilon$ and fitting a degree- $\nu$ polynomial. Figure 3 (blue circles) demonstrates how the extrapolation error decreases exponentially with the degree of the fitted polynomial.

Furthermore, we analytically solve the dependence on $\epsilon$ in the limiting case of a large number of gates and obtain the approximation

$$
\operatorname{prob}_{0}(\epsilon) \approx \operatorname{prob}_{0}-\tilde{\eta} \epsilon \frac{(1-\epsilon)^{\nu}}{2 \epsilon-1} \approx \frac{a_{1} \epsilon+a_{2} \epsilon^{2}+a_{3} \epsilon^{3}}{1+a_{4} \epsilon+a_{5} \epsilon^{2}},
$$

where $\tilde{\eta}$ is a constant. The above $(3,3)$ Padé approximation of the analytical dependence can be determined by fitting the coefficients $a_{1}, a_{2}, a_{3}, a_{4}, a_{5}$. These Padé approximations appear to slightly outperform degree- $k$ polynomial extrapolations in Fig. 3. Refer to Theorem 3 for more details.

In summary, guided by analytical arguments in example 3 we propose an efficient and straightforward approach to mitigate experimental errors that occur during the derangement circuit. Although in realistic scenarios an experimentalist may not be able to perfectly amplify all errors, we demonstrate below that extrapolation techniques can still significantly reduce the impact of noise. Note, however, that for an increasing number of qubits the noise in the controlled-SWAP gates accumulates and might attenuate the output probability prob $_{0}$. Estimating this attenuated probability at increased error rates-as required for extrapolation-requires an increased number of measurements. For example, a factor of 0.1 attenuation threshold could be approximated via the formula $0.1=(1-\epsilon)^{N(n-1)}$, and at a gate error $\epsilon=10^{-3}$ it limits the maximal number of qubits as $N(n-1) \leq 2301$ - which is still an encouraging figure in practice. We note that other error mitigation schemes could also be applied straightforwardly to address errors happening during the derangement measurement.

\section{B. Limitations of the technique}

There is one main limitation of the present approach: In a realistic experiment one can expect that coherent errors occur. As opposed to error correcting schemes, our ESD approach is completely oblivious to these and ultimately such errors will limit precision. Nevertheless, well-established techniques enable us to suppress these coherent errors, e.g., via converting them into incoherent errors by Pauli twirling [66-69]. Furthermore, as discussed above, even incoherent noise models introduce a mismatch in the dominant eigenvector which can be expressed via $\sqrt{1-c}\left|\psi_{\text {id }}\right\rangle+\sqrt{c}\left|\psi_{\text {err }}\right\rangle$. While the coherent mismatch $c$ limits the precision of the present approach, we present a comprehensive analysis and provide strong theoretical guarantees in Ref. [45] that its impact decreases when increasing the scale of the computation. See also Appendix $\mathrm{C}$ for an illustration of how this error can be mitigated.

Furthermore, the present approach is expected to be particularly well suited for variational quantum algorithms. First, the impact of coherent mismatch is guaranteed to be quadratically smaller when the aim is to prepare eigenstates [45]. Second, variational algorithms are inherently robust to this kind of error as a variational optimization implicitly minimizes the impact of coherent errors. We also remark that in the context of variational algorithms one could slightly readjust variational parameters such that the overlaps between copies $\operatorname{Tr}\left[\rho_{k} \rho_{l}\right]$ are maximal for every $k \neq l$ note that measuring such overlaps is possible with the setup in Fig. 1. This ensures us that the dominant eigenvector of every copy is (close to) identical. One could also use Clifford circuits to calibrate or validate the quantum device by comparing to expectation values obtained from (efficient) classical simulations $[18,19]$.

We further remark that we have also neglected the effect of measurement errors, i.e., when the probability of collapsing into state 0 is biased. Nevertheless, there exist well-established techniques for mitigating the effect of such imperfections [12,70].

\section{PRACTICAL APPLICATIONS}

Recall that near-term quantum devices are limited to shallow quantum circuits due to their inability to implement quantum error correction. Nevertheless, such shallow circuits may still be of high practical value as, for example, they may allow one to approximate ground-state energies of Hamiltonians $\mathcal{H}$, which cannot be estimated by other means [11-13,22-27]. Let us consider a spin-ring Hamiltonian with a constant coupling $J=0.1$ and uniformly randomly generated on-site interaction strengths $\omega_{k} \in[-1,1]$ as

$$
\mathcal{H}=\sum_{k \in \operatorname{ring}(N)} \omega_{k} Z_{k}+J \vec{\sigma}_{k} \cdot \vec{\sigma}_{k+1},
$$

for the following reasons: (a) this Hamiltonian is relevant in the context of condensed matter phenomena, such as manybody localization [71], but its ground state cannot be approximated classically for large $N$ [72,73]; (b) it has a very simple structure as well as a linearly scaling number of Pauli observables $\sigma$; (c) it is closely related to other important Hamiltonians, cf. approximate optimisation algorithms (QAOA) or spin systems in materials science $[11-13,74,75]$.

We prepare the ground state via the usual variational Hamiltonian Ansatz [11-13], which was proposed in the context of QAOA [22,74,75], but has successfully been extended to and analyzed in the context of, e.g., quantum 
chemistry, the Hubbard model, as well as spin systems [64,76-78]. It consists of alternating layers of discretized time evolutions as illustrated in Fig 4(a); refer to Appendix $\mathrm{F}$ for more details. We consider a quantum device that can natively implement single-qubit $R_{y}$ and $R_{z}$ rotation gates as well as XX gates of the form $\exp \left[-i \theta X_{j} X_{k}\right]$ between any pairs $j \neq k$ of qubits, i.e., a gate set comparable to ion-trap systems [79]. Such a platform can efficiently implement the Ansatz circuit of $l$ layers using $3 \mathrm{Nl}$ applications of the entangling gates. Using general techniques of Ref. [80] we recompile the derangement circuit into hardware-native quantum operations. Table I summarizes the number of entangling $\left(\nu_{e}\right)$ and single-qubit $\left(\nu_{s}\right)$ gates required to implement the elementary controlled-SWAP operator: we find more compact representations than previous ones $[81,82]$.

We use $l=20$ Ansatz layers such that the ground-state energy in a noise-free setting could be approximated to $\Delta E \approx 10^{-4}$ and explicitly simulate $N=6$ qubits with $n=2$ copies of the noisy computational state (equivalent of a 26-qubit pure-state simulation). We discuss in Appendix E that controlled-SWAP gates in the derangement circuit need only be recompiled up to a local SU(4) freedom as shown in Fig. 4(b); see also the second and third columns in Table I. We thus need less than $5 N=30$ entangling gates for the mitigation, which is significantly fewer than the $3 \mathrm{Nl}=360$ entangling gates required for the main computation. Figure 4(c) (red squares) shows unmitigated errors when estimating the ground-state energy of $\mathcal{H}$. We assume a noise model in which the vast majority of errors is due to dephasing and damping (relaxation), which the experimentalist can perfectly amplify. We additionally assume that a small depolarizing noise, approximately $6 \%$ of the overall gate error rate, affects the qubits that the experimentalist cannot amplify. This limits extrapolation techniques $[12,14-17,65]$ to a finite precision, as shown in Fig. 4(c) (orange diamonds). In contrast, the present approach can suppress errors under arbitrary noise models. Indeed, even with a noisy derangement circuit, one can drastically reduce errors by orders of magnitude, as shown in Fig. 4(c) (magenta dots).

As discussed above, we can apply zero-noise extrapolation to mitigate the effect of errors in the derangement circuit. As such, extrapolation in Fig. 4(c) (black crosses) can almost fully mitigate errors in the derangement circuit as black crosses approach the blue dashed line, i.e., the performance of the noiseless derangement circuit $D_{2}$. Thus it would be advantageous to prepare a larger number of

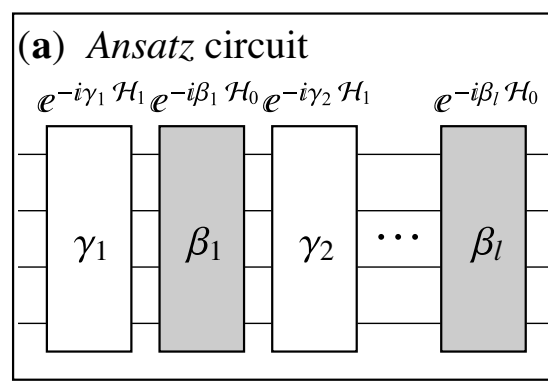

(b) Recompilation

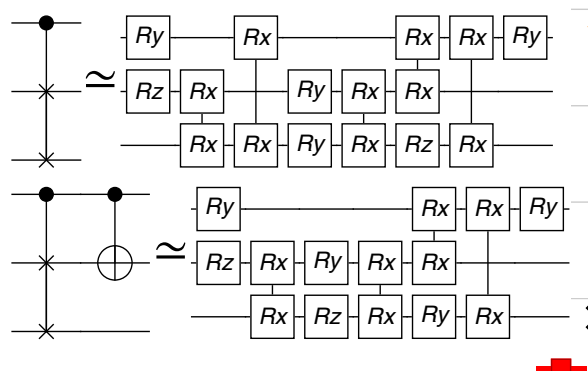

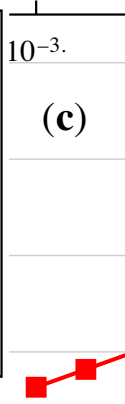

(c)

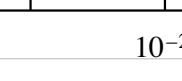

$0^{-2}$

Circuit error rate $(\xi)$

\section{$10^{-1 .}$}
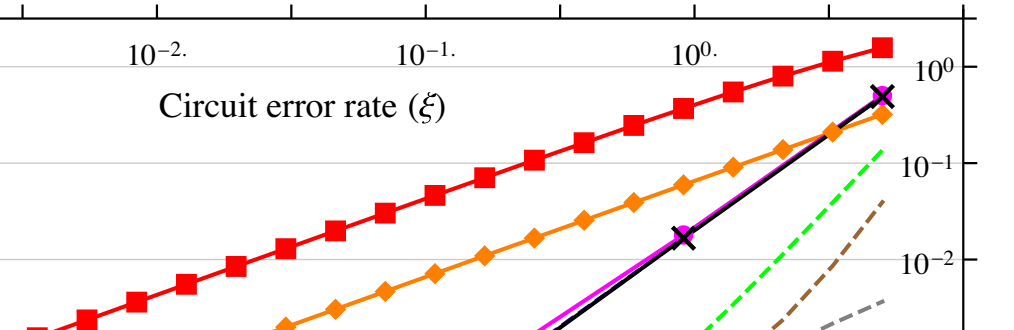
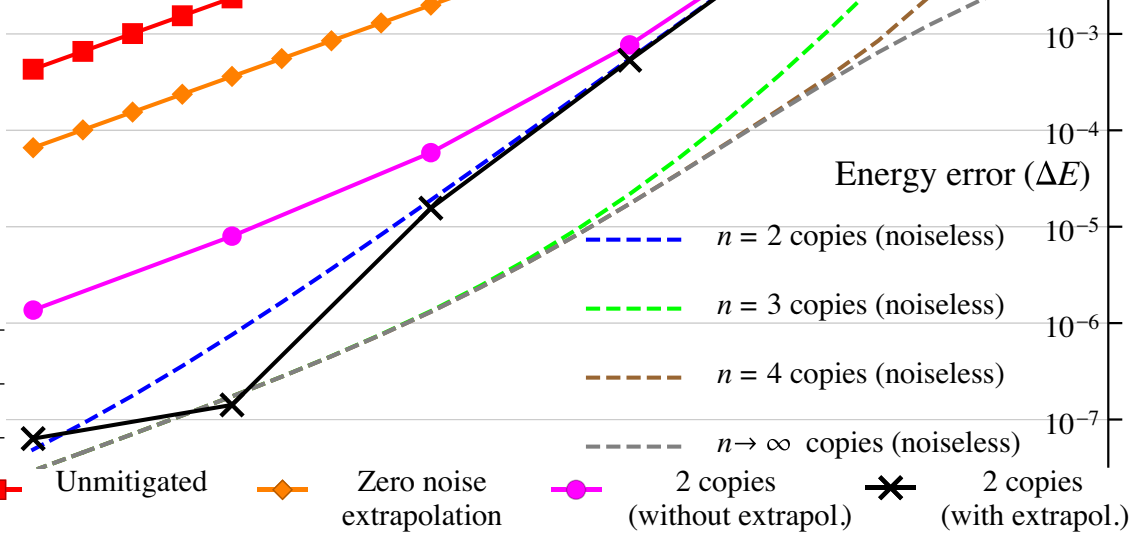

FIG. 4. (a) Ansatz used to prepare the ground state of the Hamiltonian in Eq. (8) for $N=6$ qubits. (b) Type B (type C) recompilation of controlled-SWAP (including observable) gates from Table I requires 5 (4) applications of the hardware-native entangling gates. (c) Error in estimating the ground-state energy with and without mitigation as a function of the number of expected errors $\xi$ in the Ansatz circuit. We assume that the experimentalist can amplify the vast majority of the noise (94\%) in the hardware-native gates, but not all of it, limiting extrapolation to a finite precision (orange diamonds). Although the derangement circuit is also degraded by noise, it can still drastically reduce errors both in combination with (black crosses) and without extrapolation (magenta dots). Dashed lines correspond to $\operatorname{Tr}\left[\mathcal{H} \rho^{n}\right] / \operatorname{Tr}\left[\rho^{n}\right]$ as obtained via noiseless derangement circuits. When increasing $n$, we approach in exponential order a nonzero error (dashed gray line) which is due to the coherent mismatch in the dominant eigenvector. The present demonstration on $2 \times 6+1$ qubits should rather be viewed as a worst-case scenario since increasing the scale of the computation will favor the ESD approach. 
copies $n>2$ to further suppress the errors, as illustrated in Fig. 4(c) (green and brown dashed lines). We remark, however, that going significantly beyond $n=4$ copies may not be relevant in practice for the following reasons. (a) In the practically most important region with $\xi \lesssim 1$, errors may be sufficiently suppressed below the level of other practical factors, such as shot noise, or the approximation error $\Delta E \approx 10^{-4}$ due to insufficient Ansatz depth. (b) In the limit of a large number of copies, i.e., $n \rightarrow \infty$, a constant error is approached which is due to the coherent mismatch Fig. 4(c) (gray dashed line). (c) The region with $\xi \gtrsim 2$ is practically inaccessible due to the rapidly increasing measurement overhead from result 3 via $f=\mathcal{O}\left(\ln \lambda^{-1}\right)=$ $\mathcal{O}(\xi)$ using that $\lambda=\mathcal{O}\left(e^{-\xi}\right)$ [45]. Note that it is generally the drawback of all mitigation techniques that their measurement cost grows exponentially with $\xi$ and becomes prohibitive when $\xi \gtrsim 2$ [12].

Let us finally emphasize that one should look at the present demonstration as a worst-case scenario for the following reasons. (a) Practical value is expected when computations are scaled beyond $N>20$ qubits [72,73], for which the Ansatz layers need to be increased beyond the present $l=20$, e.g., see Refs. [62,63,78]. This leads to an increasing ratio $r_{e}$ of the number of entangling gates in the main computation relative to the derangement circuit as $r_{e}=(3 / 5) l(N)$. Here the number of layers $l(N)>\mathcal{O}\left(N^{0}\right)$ needs to grow faster than a constant. (b) The impact of coherent mismatch in Fig. 4(c) (gray dashed line) is guaranteed to decrease as the number of gates increases [45]. (c) Approximating ground states of Hamiltonians other than the one in Eq. (8) may require more complex Ansatz circuits with more rapidly growing gate counts. For example, simulating the Hubbard model on $N=50$ qubits - one of the promising candidates for demonstrating practical quantum advantage-requires $\approx 2 \times 10^{4}$ entangling gates [83], while the derangement circuit requires only a few hundred, resulting in the ratio of entangling gates as $r_{e} \approx 10^{2}$. An even more pronounced example is the case of molecular Hamiltonians in which the number of Pauli terms may grow as $\mathcal{O}\left(N^{4}\right)$ [84]. (d) The Ansatz was optimized in a noiseless, pure-state simulation and reoptimizing the parameters may reduce the impact of coherent mismatch. (e) In the present case we assume 94\% of gate errors can be amplified perfectly: the experimentalist may only have control of a smaller fraction of errors further limiting the precision of extrapolation techniques.

We also consider the example of a connectivity constrained architecture in Appendix F: the number of 2-qubit gates to implement the derangement circuit is increased from $5 N$ to $6 N$, while in the Ansatz it is increased from $3 \mathrm{Nl}$ to $9 \mathrm{Nl}$. Thus, in such a scenario connectivity constraints work in our favor. Of course, in principle, specific hardware may be fabricated to optimally accommodate the present technique as well as one may utilize long-range links between macroscopically separate quantum processors [85].

\section{DISCUSSION AND CONCLUSION}

This work has introduced a novel principle for suppressing errors in near-term quantum devices. As opposed to error mitigation techniques, our ESD approach requires an increased system size: By preparing $n$ identical copies of a computational state, our derangement circuit protects its permutation symmetry and suppresses errors in an expectation value measurement exponentially (in the number $n$ of copies). Furthermore, the ESD is very NISQ friendly, since the $n$ copies of the computational state can be prepared completely independently and they only need to be "bridged" by a shallow derangement circuit immediately prior to measurement. Furthermore, the significant advantage of the ESD approach is that it is completely oblivious to the error model during the state-preparation process and works (in principle) with arbitrarily high error rates. As such, the present approach could be compared to other mitigation techniques. While quasiprobability techniques $[15,17-19]$ may in principle be able to perfectly negate the effect of errors, they require an exponentially growing number of circuit variants together with a perfect knowledge of the error model. Any deviation from the assumed noise model results in errors, which may grow exponentially with the number of gates. Symmetry verification is another successful mitigation technique that could be used if exploitable symmetries are present [20,86]; however, it cannot reduce errors that fall within the subspace of appropriate symmetry. Furthermore, zero-noise extrapolation $[12,14-17,65]$ can in principle be applied generally; however, the experimentalist may not be able to perfectly amplify all errors; see Fig. 4(c). In contrast, the present approach can be applied completely generally in any scenario. Note, however, that these existing mitigation techniques will be highly relevant as they can be used in combination with the present approach, as demonstrated above.

The main limitation of the ESD approach is that it cannot address coherent noise or a coherent mismatch in the dominant eigenvector, although those errors can be exponentially smaller than the incoherent decay of the fidelity and are guaranteed to decrease when increasing the scale of the computation [45]. As long as the derangement circuit is assumed to be perfect, the sample complexity of our ESD approach is polynomial in the inverse precision $\mathcal{E}^{-1}$ and comparable to the standard shot-noise limit in practically relevant scenarios, i.e., when the number of expected errors in the main computation is below $\xi \approx 1$. Errors during the derangement process do degrade the performance of the present approach and one needs to rely on error mitigation techniques to reduce this impact. Nevertheless, it was shown above that the number of gates in the derangement 
circuit is expected to become negligible relative to the main computation when scaling up computations.

Let us now briefly comment on prior approaches that similarly consider identical copies of quantum states and similarly apply SWAP operators (or generalizations thereof). In fact, numerous prior works have considered and exploited the permutation symmetry of identical copies of mixed states in the context of, e.g., reconstructing spectral properties of mixed quantum states [47-53], probing their entanglement characteristics [87-89], for constructing universal quantum software [90], and for optimal state discrimination [91-93]. Indeed, in the special case of $n=2$ copies, our scheme is comparable to a modification of the usual SWAP-test circuit [47]. However, as opposed to previous works, here we are not interested in the input mixed state $\rho$, but only in its dominant eigenvector $|\psi\rangle$ that represents a computational quantum state. In fact, we regard any other contribution in the state $\rho$ as "noise" which we aim to exclude from the expectationvalue measurement process. The present approach could also be compared to entanglement distillation protocols [94-96]; however, our derangement circuit cannot exponentially improve the "quality" of the input states, but only exclude erroneous contributions from the expectation-value measurement process.

Let us finally remark that the ESD approach leaves a lot of room for a large number of different physical implementations, beyond the circuit in Fig. 1 that has been analyzed in detail in this work. Our circuit in Fig. 1 is only one possible realization of the general principle outlined here and even this circuit has a large number of invariants. We only need to remark here that the results presented here are very general, and our example circuit could certainly be improved by combining it with advanced techniques, for example, by simultaneously measuring groups of commuting observables $[43,44]$ — but we expect these can only introduce constant factor improvements and will not change the main results in this work. In future work we will explore the numerous possibilities offered by the general principle introduced here.

Please also refer to the online repository [58] for simulation and demonstration material.

\section{ACKNOWLEDGMENTS}

I would like to thank Simon C. Benjamin for his invaluable comments and challenging questions. His help and support was crucial for finalizing this work. I would like to thank Earl Campbell, Robert Zeier, Suguru Endo, and Ying $\mathrm{Li}$ for their very constructive and valuable comments on drafts of this work. I acknowledge funding received from EU H2020-FETFLAG-03-2018 under Grant Agreement No. 820495 (AQTION) and the QCS Hub (EPSRC Hub Grant under the Agreement No. EP/T001062/
1) for support including hardware provision. The numerical modeling involved in this study made use of the Quantum Exact Simulation Toolkit (QuEST), and the recent development QuESTlink [97], which permits the user to use Mathematica as the integrated front end. I am grateful to those who have contributed to both of these valuable tools.

\section{Note added}

Recently, a related paper appeared that proposes a very similar idea [98], while mostly focusing on the $n=2$ scenario. The main difference is that Ref. [98] uses the trace distance to quantify errors thereby also taking into account the effect of coherent mismatch. In contrast, in result 1 I only quantify errors with respect to the dominant eigenvector, while I defer a comprehensive analysis of the coherent mismatch to the subsequent paper [45] for the following reasons.

(a) Reference [98] numerically computed and plotted the trace distance in a comprehensive range of scenarios, and noted that the bound is "pessimistic" as it overestimates errors. As such, in Ref. [45] I show that in most practical scenarios this trace distance should not be used since a quadratically smaller bound exists, i.e., the square $c$ of the trace distance $\sqrt{c}$. This is nicely illustrated in Fig. 4(c) (gray dashed line): at a circuit error rate $\xi=0.1$ the actual error is $\Delta E \approx 7.5 \times 10^{-6}$ while the bound of Ref. [98] is misleading as it is orders of magnitude larger $2 \sqrt{c}\|\mathcal{H}\|_{\infty}=9.2 \times 10^{-3}$. The relation between the two bounds is discussed in more detail in Ref. [45].

(b) Going beyond the pessimistic bound of Ref. [98] and realistically characterizing the coherent mismatch is a very complex problem as it is related to important themes in mathematics, such as Weyl's inequalitiessolving of which was a major breakthrough. In Ref. [45] I provide strong theoretical guarantees that the coherent mismatch can be exponentially small and decreases when increasing the scale of the computation. Thus, practitioners need principally care about the errors with respect to measuring expectation values in the dominant eigenvector, cf. Fig. 4.

(c) It is also interesting to note that result 1 only depends on spectral properties, i.e., eigenvalues $\lambda_{k}$ and Rényi entropies $H_{n}$, that may be estimated experimentally. In contrast, estimating the trace distance of Ref. [98] would require one to prepare the ideal, perfect, noiseless quantum state as well as the state $\rho^{n} / \operatorname{Tr}\left[\rho^{n}\right]$, which is prohibitive.

Other studies have already reported ideas extending or varying these original concepts. For example, Ref. [99] introduces a generalization of the presented permutationsymmetry principles. Furthermore, Ref. [100] proposes that the derangement circuit $D_{n}:=\mathrm{SWAP}_{1, n} \cdots \mathrm{SWAP}_{1,3} \mathrm{SWAP}_{1,2}$ can be realized in a qubit-efficient manner by utilizing qubit 
resets, thus drastically reducing resource requirements of the present approach.

\section{APPENDIX A: DERANGEMENT MEASUREMENTS AND SUPPRESSING ERRORS}

In this Appendix, we prove that the derangement circuit in Fig. 1 can be used to estimate expectation values. We then prove upper bounds on approximation errors with or without using Rényi entropies of quantum states. We finally prove sample complexities of our ESD approach.

Definition 1.-We define the set $\mathfrak{D}_{n}$ of derangement operators that permute $n \geq 2$ quantum registers via their unitary representation as

$$
\begin{aligned}
\text { every } D_{n} & \in \mathfrak{D}_{n}, \\
\text { such that } D_{n}\left|\psi_{1}, \psi_{2}, \ldots, \psi_{n}\right\rangle & =\left|\psi_{s(1)}, \psi_{s(2)}, \ldots, \psi_{s(n)}\right\rangle .
\end{aligned}
$$

Here all $s \in S_{n}$ are permutations of the index set $\{1,2, \ldots, n\}$ with no fixed point; i.e., $s$ are derangements $[56,57]$. Here $S_{n}$ denotes the symmetric group. For $n \geq 4$ we also demand that $s$ are $n$-cycles (standard cyclic permutations of maximal length [57]), which are a subset of derangements. The number of unique ( $n$-cycle) derangement operators is given as $\left|\mathfrak{D}_{n}\right|=(n-1)$ !. Because of seminal results of Dénes, $s$ can be decomposed into $n-1$ transpositions [59] and therefore $D_{n}$ decomposes into $n-1$ pairwise SWAP operators of the quantum registers. One can therefore construct minimal SWAP circuits by (bijectively) mapping the corresponding permutations performed by $D_{n} \in \mathfrak{D}_{n}$ to graph trees.

Theorem 1.-We consider $n$ identical copies of the same quantum register $\rho$ in a separable state as $\rho^{\otimes n}$. Methods A and B, as illustrated in Fig. 1, result in the probability of measuring the ancilla in the 0 state as

$$
\text { Method A or B : } \operatorname{prob}_{0}=\frac{1}{2}+\frac{1}{2} \operatorname{Tr}\left[\rho^{n} \sigma\right] \text {. }
$$

Here $\sigma$ is a unitary (Hermitian) observable (or otherwise the real part of a unitary operator is estimated).

Proof.-We start by recapitulating that any density operator $\rho$ admits the following spectral decomposition (note that here we use a different notation than what in the main text),

$$
\begin{aligned}
\rho= & \sum_{k=1}^{2^{N}} p_{k}\left|\psi_{k}\right\rangle\left\langle\psi_{k}\right| ; \quad \text { thus } \\
\rho^{\otimes n}= & \sum_{k_{1}, k_{2}, \ldots k_{n}=1}^{2^{N}} p_{k_{1}} p_{k_{2}} \cdots p_{k_{n}}\left|\psi_{k_{1}}, \psi_{k_{2}}, \ldots, \psi_{k_{n}}\right\rangle \\
& \times\left\langle\psi_{k_{1}}, \psi_{k_{2}}, \ldots, \psi_{k_{n}}\right|,
\end{aligned}
$$

where the second equation is the spectral decomposition of $n$ copies of the same state.

Recall that the action of any unitary circuit $U$ on a density matrix $U \rho U^{\dagger}$ represents a probabilistic mixture of its transformed eigenvectors $U\left|\psi_{k}\right\rangle$ that occur with probabilities $p_{k}$. Similarly, the action of a unitary circuit on the composite state $\rho^{\otimes n}$ can be written as a probabilistic mixture of the pure states $U\left|\psi_{k_{1}}, \psi_{k_{2}}, \ldots, \psi_{k_{n}}\right\rangle$ that occur with probabilities as products $p_{k_{1}} p_{k_{2}} \cdots p_{k_{n}}$.

Let us now derive the action of the unitary circuit in Fig. 1 on the composite quantum system $\rho^{\otimes n}$. Our proof works with any derangement operator $D_{n}$ from Definition 1, but here we only need to consider one example: we consider a cyclic shift (as originally proposed in Ref. [47]) of the registers via its explicit action on pure states as

$$
D_{n}\left|\psi_{1}, \psi_{2}, \ldots, \psi_{n}\right\rangle=\left|\psi_{n}, \psi_{1}, \ldots, \psi_{n-1}\right\rangle .
$$

Our controlled derangement operator acts on the pure state $\left|0, \psi_{k_{1}}, \psi_{k_{2}}, \ldots, \psi_{k_{n}}\right\rangle$ that occurs with a probability $p_{k_{1}} p_{k_{2}} \cdots p_{k_{n}}$, and we denote as 0 the state of the additional ancilla qubit. Applying the sequence of gates from Fig. 1 yields the following transformations of the pure states:

$$
\begin{gathered}
\left|0, \psi_{k_{1}}, \psi_{k_{2}}, \ldots, \psi_{k_{n}}\right\rangle \\
\downarrow H \\
\left(\left|1, \psi_{k_{1}}, \psi_{k_{2}}, \ldots, \psi_{k_{n}}\right\rangle+\left|0, \psi_{k_{1}}, \psi_{k_{2}}, \ldots, \psi_{k_{n}}\right\rangle\right) / \sqrt{2} \\
\downarrow \text { controlled } D_{n} \\
\left(\left|1, \psi_{k_{n}}, \psi_{k_{1}}, \ldots, \psi_{k_{n-1}}\right\rangle+\left|0, \psi_{k_{1}}, \psi_{k_{2}}, \ldots, \psi_{k_{n}}\right\rangle\right) / \sqrt{2} \\
\downarrow \text { controlled } \sigma \\
\left(\left|1, \sigma \psi_{k_{n}}, \psi_{k_{1}}, \ldots, \psi_{k_{n-1}}\right\rangle+\left|0, \psi_{k_{1}}, \psi_{k_{2}}, \ldots, \psi_{k_{n}}\right\rangle\right) / \sqrt{2} \\
\downarrow H \\
\left(\left|0, \psi_{k_{n}}, \psi_{k_{1}}, \ldots, \psi_{k_{n-1}}\right\rangle+\left|0, \psi_{k_{1}}, \psi_{k_{2}}, \ldots, \psi_{k_{n}}\right\rangle\right) / 2+\cdots
\end{gathered}
$$

It is now straightforward to show that the probability of measuring the ancilla qubit in state 0 is

$$
\begin{aligned}
\operatorname{prob}_{0}= & \frac{1}{2}+\frac{1}{2} \sum_{k_{1}, k_{2}, \ldots k_{n}=1}^{2^{N}} p_{k_{1}} p_{k_{2}} \ldots \\
& \times p_{k_{n}}\left\langle\psi_{k_{1}}, \psi_{k_{2}}, \ldots, \psi_{k_{n}} \mid \sigma \psi_{k_{n}}, \psi_{k_{1}}, \ldots, \psi_{k_{n-1}}\right\rangle,
\end{aligned}
$$

where we can simplify the inner products as

$$
\begin{aligned}
& \left\langle\psi_{k_{1}}, \psi_{k_{2}}, \ldots, \psi_{k_{n}} \mid \sigma \psi_{k_{n}}, \psi_{k_{1}}, \ldots, \psi_{k_{n-1}}\right\rangle \\
& \quad=\left\langle\psi_{k_{1}} \mid \sigma \psi_{k_{n}}\right\rangle\left\langle\psi_{k_{2}} \mid \psi_{k_{1}}\right\rangle \cdots\left\langle\psi_{k_{n}} \mid \psi_{k_{n-1}}\right\rangle \\
& \quad=\left\langle\psi_{k_{1}} \mid \sigma \psi_{k_{n}}\right\rangle \delta_{k_{2} k_{1}} \cdots \delta_{k_{n} k_{n-1}},
\end{aligned}
$$

and we have used the orthogonality of the eigenstates $\left|\psi_{k}\right\rangle$ and $\delta_{a b}$ is the Kronecker delta symbol. At this point we remark that our proof works with any derangement operator 
from Definition 1 since these will conserve the above orthonormality relation. We remark here that the corresponding permutations can be mapped to graph trees, which related to the pairs of indexes $\delta_{a b}$ in the Kronecker delta symbols in the above equation.

Substituting the above results back we obtain the expression for the ancilla probability by using that only terms with coinciding indexes contribute to the sum via $k_{1}=k_{2}=\cdots k_{n}$ :

$$
\operatorname{prob}_{0}=\frac{1}{2}+\frac{1}{2} \sum_{k=1}^{2^{N}} p_{k}^{n}\left\langle\psi_{k}|\sigma| \psi_{k}\right\rangle=\frac{1}{2}+\frac{1}{2} \operatorname{Tr}\left[\rho^{n} \sigma\right] .
$$

Example 1: Using our definition of experimental quantum states from Eq. (1), our circuit in Fig. 1 can estimate the expectation value:

$$
\operatorname{Tr}\left[\rho^{n} \sigma\right]=\lambda^{n}\langle\psi|\sigma| \psi\rangle+(1-\lambda)^{n} \sum_{k=2}^{2^{N}} p_{k}^{n}\left\langle\psi_{k}|\sigma| \psi_{k}\right\rangle .
$$

It is clear that the error probabilities $p_{k}$ are suppressed exponentially via $p_{k}^{n}$, but the dominant term gets slightly attenuated too via $\lambda^{n}$. For example, let us assume that our dominant eigenvalue is $\lambda=0.8$ and we have a high-entropy error in a subspace spanned by 100 eigenvectors via the uniform distribution $p_{k}=(1-\lambda) / 100$ when $k \leq 101$ and $p_{k}=0$ when $k>101$. We then obtain the estimate:

$$
\operatorname{Tr}\left[\rho^{n} \sigma\right]=0.8^{n}\langle\psi|\sigma| \psi\rangle+\sum_{k=2}^{101}\left(2 \times 10^{-3}\right)^{n}\left\langle\psi_{k}|\sigma| \psi_{k}\right\rangle .
$$

Since $\left|\left\langle\psi_{k}|\sigma| \psi_{k}\right\rangle\right| \leq 1$, we can upper bound the errors in $\operatorname{Tr}\left[\rho^{n} \sigma\right]=0.512\langle\psi|\sigma| \psi\rangle+E \quad$ for, $\quad$ e.g., $\quad n=3, \quad$ as $|E| \leq 8 \times 10^{-7}$. Hence our error contribution is at least 640000 times smaller than the desired expectation value. This high degree of error suppression is due to the large $n=3$ Rényi entropy of the error probabilities $p_{k}$ as

$$
\begin{aligned}
H_{3}(\underline{p}) & =-\frac{1}{2} \ln \left[\sum_{k=2}^{2^{N}} p_{k}^{n}\right]=-\frac{1}{2} \ln \left[\sum_{k=2}^{101}\left(10^{-2}\right)^{3}\right] \\
& \approx-\frac{1}{2} \ln \left[10^{-4}\right] \approx 4.6 .
\end{aligned}
$$

We will show in Theorem 2 and in Lemma 1 that the efficiency of the error suppression depends exponentially on this Rényi entropy.

Indeed, in order to obtain an accurate estimate of $\langle\psi|\sigma| \psi\rangle$ we need to have a good knowledge of the largest eigenvalue of the density matrix $\lambda$ that divides $\langle\psi|\sigma| \psi\rangle$. We assume in method B in Theorem 2 that this eigenvalue is known precisely. However, in method A we just replace our observable $\sigma$ with the identity in Fig. 1 and we directly approximate the $n$th power of the dominant eigenvalue $\lambda$ as

$$
\operatorname{Tr}\left[\rho^{n}\right]=0.8^{n}+\sum_{k=2}^{101}\left(2 \times 10^{-3}\right)^{n}
$$

for $n=3$ we obtain the result as $0.8^{3}+8 \times 10^{-7}=0.512001$, which is a very good estimate of $0.8^{3}=0.512$ as the error is 640000 times smaller than the ideal value.

Example 2: We consider now the worst-case scenario of 0-entropy error distributions. For example, let us consider the state $\rho=\lambda|\psi\rangle\left\langle\psi|+(1-\lambda)| \psi_{\text {err }}\right\rangle\left\langle\psi_{\text {err }}\right|$, which is a mixture of the ideal state $|\psi\rangle$ that occurs with a probability $\lambda$ and an erroneous state $\left|\psi_{\text {err }}\right\rangle$ which occurs with a probability $(1-\lambda)$. The error probability distribution from Eq. (1) is obtained as $p_{2}=1$ and $p_{k}=0$ for $k>2$. It follows that the error distribution has a 0 entropy and our approach completely breaks down when $\lambda \leq 1 / 2$ since the dominant eigenvector then becomes $\left|\psi_{\text {err }}\right\rangle$. We can show that the errors in the expectation value are still exponentially suppressed, but much less efficiently than before in example 1. Let us set $\lambda=0.8$ and

$$
\operatorname{Tr}\left[\rho^{n} \sigma\right]=0.8^{n}\langle\psi|\sigma| \psi\rangle+0.2^{n}\left\langle\psi_{\mathrm{err}}|\sigma| \psi_{\mathrm{err}}\right\rangle .
$$

For $n=3 \quad$ we obtain $\operatorname{Tr}\left[\rho^{3} \sigma\right]=0.512\langle\psi|\sigma| \psi\rangle+$ $0.008\left\langle\psi_{\text {err }}|\sigma| \psi_{\text {err }}\right\rangle$, and therefore the error is suppressed by a factor of 64 . This is significantly lower that the factor of 640000 suppression from example 1, which assumed a high-entropy error distribution.

\section{APPENDIX B: EXPONENTIALLY DECREASING UPPER BOUNDS ON APPROXIMATION ERRORS}

Theorem 2.-We use methods A and B from Fig. 1 to estimate the probability $\operatorname{prob}_{0}=\frac{1}{2}+\frac{1}{2} \operatorname{Tr}\left[\rho^{n} \sigma\right]$ of the ancilla qubit. In method A we use the same technique via $\sigma=\mathrm{Id}$ to estimate the probability $\operatorname{prob}_{0}^{\prime}=\frac{1}{2}+\frac{1}{2} \operatorname{Tr}\left[\rho^{n}\right]$, and our method A yields the approximation

$$
\text { Method A: } \frac{2 \operatorname{prob}_{0}-1}{2 \operatorname{prob}_{0}^{\prime}-1}=\frac{\operatorname{Tr}\left[\rho^{n} \sigma\right]}{\operatorname{Tr}\left[\rho^{n}\right]}=\langle\psi|\sigma| \psi\rangle+\mathcal{E}_{A} .
$$

In method B we assume that the largest eigenvalue $\lambda$ of the state $\rho$ is known, and therefore we have the approximation

Method B : $\left(2 \operatorname{prob}_{0}-1\right) / \lambda^{n}=\operatorname{Tr}\left[\rho^{n} \sigma\right] / \lambda^{n}=\langle\psi|\sigma| \psi\rangle+\mathcal{E}_{B}$.

The approximation errors are bounded via $\left|\mathcal{E}_{A}\right| \leq\left(2 Q_{n}\right) /$ $\left(1+Q_{n}\right)$ and $\left|\mathcal{E}_{B}\right| \leq Q_{n}$, and we prove in Lemma 1 that the bounding sequence $Q_{n}=\left(\lambda^{-1}-1\right)^{n}\|\underline{p}\|_{n}^{n}$ generally decays exponentially when we increase $n$ or when we increase the Rényi entropy of the probability vector $p$.

Proof-Let us recapitulate the explicit form of the density matrix from Eq. (1) as 


$$
\rho=\lambda|\psi\rangle\left\langle\psi\left|+(1-\lambda) \sum_{k=2}^{2^{N}} p_{k}\right| \psi_{k}\right\rangle\left\langle\psi_{k}\right| .
$$

We can evaluate the expressions for the trace operation as $\operatorname{Tr}\left[\rho^{n} \sigma\right]=\lambda^{n}\langle\psi|\sigma| \psi\rangle+(1-\lambda)^{n} \sum_{k=2}^{2^{N}} p_{k}^{n}\left\langle\psi_{k}|\sigma| \psi_{k}\right\rangle \quad$ and $\left|\sum_{k=2}^{2^{N}} p_{k}^{n}\left\langle\psi_{k}|\sigma| \psi_{k}\right\rangle\right| \leq \sum_{k=2}^{2^{N}} p_{k}^{n}=\|\underline{p}\|_{n}^{n}$,

where we have used that $\left|\left\langle\psi_{k}|\sigma| \psi_{k}\right\rangle\right| \leq 1$ due to unitarity of $\sigma$ and $\|\underline{p}\|_{n}$ is the $n$-norm of the probability vector $\underline{p}$.

Method B: Here our aim is to estimate $\operatorname{Tr}\left[\rho^{n} \sigma\right]$ and $\lambda^{n}$ is known exactly. The error term can be calculated via

$$
\begin{aligned}
\left|\mathcal{E}_{B}\right| & =\left|\frac{\operatorname{Tr}\left[\rho^{n} \sigma\right]}{\lambda^{n}}-\langle\psi|\sigma| \psi\rangle\right|=\frac{(1-\lambda)^{n}}{\lambda^{n}}\left|\sum_{k=2}^{2^{N}} p_{k}^{n}\left\langle\psi_{k}|\sigma| \psi_{k}\right\rangle\right| \\
& \leq \frac{(1-\lambda)^{n}}{\lambda^{n}}\|\underline{p}\|_{n}^{n}=: Q_{n},
\end{aligned}
$$

and here we have defined the sequence $Q_{n}$.

Method A: In this case we estimate $\operatorname{Tr}\left[\rho^{n} \sigma\right]$ and $\operatorname{Tr}\left[\rho^{n}\right]$, and we now calculate the error term using that $\operatorname{Tr}\left[\rho^{n}\right]=$ $\lambda^{n}+(1-\lambda)^{n} \sum_{k} p_{k}^{n}=\lambda^{n}+(1-\lambda)^{n}\|\underline{p}\|_{n}^{n}$. Indeed, we obtain

$$
\begin{aligned}
\left|\mathcal{E}_{A}\right| & =\left|\frac{\operatorname{Tr}\left[\rho^{n} \sigma\right]}{\operatorname{Tr}\left[\rho^{n}\right]}-\langle\psi|\sigma| \psi\rangle\right| \\
& =\left|\frac{\langle\psi|\sigma| \psi\rangle+\lambda^{-n}(1-\lambda)^{n} \sum_{k=2}^{2^{N}} p_{k}^{n}\left\langle\psi_{k}|\sigma| \psi_{k}\right\rangle}{1+\lambda^{-n}(1-\lambda)^{n}|| \underline{p}||_{n}^{n}}-\langle\psi|\sigma| \psi\rangle\right| \\
& =\left|\frac{\langle\psi|\sigma| \psi\rangle+Z}{1+Q_{n}}-\langle\psi|\sigma| \psi\rangle\right|,
\end{aligned}
$$

where we have used the notation $Z=\lambda^{-n}(1-\lambda)^{n} \times$ $\sum_{k=2}^{2^{N}} p_{k}^{n}\left\langle\psi_{k}|\sigma| \psi_{k}\right\rangle$ for simplicity. It follows that the error term is bounded via

$$
\begin{aligned}
\left|\mathcal{E}_{A}\right| & =\left|\frac{\langle\psi|\sigma| \psi\rangle+Z}{1+Q_{n}}-\langle\psi|\sigma| \psi\rangle\right| \\
& =\left|\frac{Z-Q_{n}\langle\psi|\sigma| \psi\rangle}{1+Q_{n}}\right| .
\end{aligned}
$$

Let us now upper bound this expression as

$$
\begin{aligned}
& \left(1+Q_{n}\right)^{-1}\left|Z-Q_{n}\langle\psi|\sigma| \psi\rangle\right| \\
& \quad \leq\left(1+Q_{n}\right)^{-1}\left[|Z|+Q_{n}|\langle\psi|\sigma| \psi\rangle|\right],
\end{aligned}
$$

where we have used the triangle inequality as $|a-b| \leq|a|+|b|$. We can now use from before that $|Z| \leq Q_{n}$, which results in the error term:

$$
\begin{aligned}
\left|\mathcal{E}_{A}\right| & \leq \frac{Q_{n}+Q_{n}|\langle\psi|\sigma| \psi\rangle|}{1+Q_{n}}=\frac{Q_{n}}{1+Q_{n}}(1+|\langle\psi|\sigma| \psi\rangle|) \\
& \leq \frac{2 Q_{n}}{1+Q_{n}} .
\end{aligned}
$$

This concludes our proof.

Lemma 1.- The sequence $Q_{n}$ in our upper bounds in Theorem 2 decreases exponentially for a fixed $n$ when we increase the Rényi entropy $H_{n}(\underline{p})$ of the error probability vector $\underline{p}$ from Eq. (1) as $Q_{n}=\left(\lambda^{-1}-1\right)^{n} \exp \left[-(n-1) H_{n}(\underline{p})\right]$. Furthermore, the sequence generally decays exponentially via $Q_{n} \leq\left(p_{\max }\right)^{-1} Q^{n}$, where we define the suppression factor $Q:=\left(\lambda^{-1}-1\right) p_{\max }<1$ and $p_{\max }$ is the largest error probability from Eq. (1).

Proof.-The first part of the proof straightforwardly follows by substituting the expression for the Rényi entropy [46],

$$
H_{n}(\underline{p})=\frac{1}{1-n} \ln \left[\sum_{k=2}^{2^{N}} p_{k}^{n}\right]=\frac{n}{1-n} \ln \|\underline{p}\|_{n},
$$

into the expression for $Q$ as

$$
\begin{aligned}
Q_{n} & =\left[\left(\lambda^{-1}-1\right)\|\underline{p}\|_{n}\right]^{n}=\left(\lambda^{-1}-1\right)^{n} \exp \left[-\frac{n-1}{n} H_{n}(\underline{p})\right]^{n} \\
& =\left(\lambda^{-1}-1\right)^{n} \exp \left[-(n-1) H_{n}(\underline{p})\right] .
\end{aligned}
$$

This concludes the first part of our proof.

Let us now prove that the sequence $Q_{n}$ decreases in exponential order when we increase $n$. Using the wellknown series of inequalities satisfied by the Rényi entropies as $H_{\infty}(\underline{p}) \cdots \leq H_{n}(\underline{p}) \leq H_{n-1}(\underline{p}) \leq \cdots \leq H_{1}(\underline{p})$, we obtain the general bound $-\ln p_{\max } \leq H_{n}(\underline{p})$ for all $n$, and we define the largest error probability $p_{\max }:=\max _{k} p_{k}$. It follows that

$$
\begin{aligned}
Q_{n} \leq\left(\lambda^{-1}-1\right)^{n}\left(p_{\max }\right)^{n-1} & =\left(p_{\max }\right)^{-1}\left[\left(\lambda^{-1}-1\right) p_{\max }\right]^{n} \\
& =:\left(p_{\max }\right)^{-1} Q^{n} .
\end{aligned}
$$

The upper bound $Q<1$ holds due to our condition below Eq. (1) as $(\lambda-1) p_{k}<\lambda$ for every probability $k=\left\{2,3, \ldots, 2^{N}\right\}$. It follows that $p_{\max }<\left(\lambda^{-1}-1\right)^{-1}$ and therefore $Q=\left(\lambda^{-1}-1\right) p_{\max }<1$.

Lemma 2.-Determining the expectation value $\langle\psi|\sigma| \psi\rangle$ from Theorem 2 to a fixed precision $\mathcal{E}$ requires $n=$ $\ln \mathcal{E}^{-1}+\ln \left[2\left(p_{\max }\right)^{-1}\right] / \ln Q^{-1}$ copies of the quantum state $\rho$ (one needs to apply the ceiling function to round this up to the nearest integer). Here $Q<1$ is the suppression factor from Theorem 2 and from Lemma 1.

Reducing shot noise to the desired precision $\mathcal{E}$ requires the following number of samples. In method $\mathrm{A}$ one needs to assign $N_{s, 1}$ samples to determine prob ${ }_{0}$ and $N_{s, 2}$ samples to determine $\operatorname{prob}_{0}^{\prime}$ as 
Method A: $\quad N_{s, 1}=\mathcal{O}\left[\mathcal{E}^{-2(1+f)}\right]=\operatorname{poly}\left(\mathcal{E}^{-1}\right) \quad$ and

$$
N_{s, 2}=\mathcal{O}\left[\mathcal{E}^{-2(1+2 f)}\right]=\operatorname{poly}\left(\mathcal{E}^{-1}\right) .
$$

The overall number of measurements required is $N_{s}=$ $N_{s, 1}+N_{s, 2}=\mathcal{O}\left[\mathcal{E}^{-2(1+2 f)}\right]$. In method B one only needs to determine $\operatorname{prob}_{0}$ since $\lambda^{n}$ is known. The number of samples scales as

$$
\text { Method B : } N_{s}=\mathcal{O}\left[\mathcal{E}^{-2(1+f)}\right]=\operatorname{poly}\left(\mathcal{E}^{-1}\right) .
$$

Indeed, in both cases the measurement cost grows polynomially with the inverse precision $\mathcal{E}^{-1}$ and its polynomial order is determined by $f:=\ln \left(\lambda^{-1}\right) / \ln \left(Q^{-1}\right)$. The standard shot-noise limit $\mathcal{E}^{-2}$ is only slightly modified by $f$ in the case of good quality quantum states or in the case of highentropy probabilities.

Proof.-Let us first compute the upper bound on the number of copies $n$ required to achieve a fixed precision $\mathcal{E} \ll 1$. We use the upper bounds from Theorem 2 as $\left|\mathcal{E}_{A}\right| \leq \mathcal{E}=\left(2 Q_{n}\right) /\left(1+Q_{n}\right) \approx 2 Q_{n}$ and $\left|\mathcal{E}_{B}\right| \leq \mathcal{E}=Q_{n}$. It is clear that the precision of method A differs by a factor of 2 for $\mathcal{E} \ll 1$, and we use this expression for both methods for simplicity. Let us use the exponentially decreasing upper bounds on $Q_{n}$ from Lemma 1 and write $\left|\mathcal{E}_{A}\right| \leq$ $2\left(p_{\max }\right)^{-1} Q^{n}$, and $\left|\mathcal{E}_{B}\right| \leq 2\left(p_{\max }\right)^{-1} Q^{n}$, where we have defined the suppression factor as $Q:=\left(\lambda^{-1}-1\right) p_{\max }<1$. It is straightforward to express $n$ as

$$
n=\frac{\ln \mathcal{E}^{-1}+\ln \left[2\left(p_{\max }\right)^{-1}\right]}{\ln Q^{-1}} .
$$

Remark: Let us further expand the above equation by using our expression from Lemma 1 as $Q_{n}=$ $\left(\lambda^{-1}-1\right)^{n} \exp \left[-(n-1) H_{n}(\underline{p})\right]$, which results in

$$
\begin{aligned}
-\ln \left(\mathcal{E}^{-1}\right) & =\ln \mathcal{E}=\ln 2 Q_{n} \\
& =\ln (2)+n \ln \left[\left(\lambda^{-1}-1\right)\right]-(n-1) H_{n}(\underline{p}) \\
& =\ln (2)+H_{n}(\underline{p})+n\left\{\ln \left[\left(\lambda^{-1}-1\right)\right]-H_{n}(\underline{p})\right\} .
\end{aligned}
$$

We can express $n$ as

$$
n=\frac{\ln \left(\mathcal{E}^{-1}\right)+H_{n}(\underline{p})+\ln (2)}{H_{n}(\underline{p})-\ln \left[\left(\lambda^{-1}-1\right)\right]}=\mathcal{O}\left[\ln \left(\mathcal{E}^{-1}\right)\right] .
$$

We remark that the denominator is positive due to the bound on Rényi entropies from Lemma 1 as $\ln \left[\left(\lambda^{-1}-1\right)\right]<$ $H_{n}(\underline{p})$. One should actually use the ceil function to round up the right-hand expression to the nearest integer. Note that the above expression implicitly depends on $n$ via the Rényi entropy $H_{n}(\underline{p})$, but one could always use the series of inequalities $0 \leq H_{n}(\underline{p}) \leq H_{n-1}(\underline{p}) \leq \cdots H_{2}(\underline{p}) \leq$ $H_{1}(\underline{p})$ to bound the value of $n$. It is straightforward to show now that in the limiting scenarios $H_{2}(p) \gg 1$ or $\lambda \approx 1$ we recover $n \rightarrow 1$ (via the ceil function). Let us now express the scaling with respect to shot noise.

Method B: We estimate the probability prob $_{0}$ from Theorem 2 and we exactly know $\lambda^{n}$. Our precision $\mathcal{E}$ is determined by the variance of our estimator which can be obtained as

$$
\begin{aligned}
\mathcal{E}^{2} & =\operatorname{Var}[\langle\psi|\sigma| \psi\rangle]=\operatorname{Var}\left[\frac{2 \operatorname{prob}_{0}-1}{\lambda^{n}}\right]=\frac{4 \operatorname{Var}\left[\operatorname{prob}_{0}\right]}{\lambda^{2 n}} \\
& =\frac{4 \operatorname{prob}_{0}\left(1-\operatorname{prob}_{0}\right)}{N_{s} \lambda^{2 n}},
\end{aligned}
$$

where we have used that the variance of the binomial distribution is $\operatorname{prob}_{0}\left(1-\operatorname{prob}_{0}\right) / N_{s}$ and $N_{s}$ is the number of samples. We can explicitly express the number of shots $N_{s}$ required to reach a fixed precision $\mathcal{E}$ as

$$
N_{s}=\frac{4 \operatorname{prob}_{0}\left(1-\operatorname{prob}_{0}\right)}{\mathcal{E}^{2} \lambda^{2 n}} .
$$

Let us now simplify $\lambda^{2 n}$ by expressing the dependence of $n$ on the precision above $\mathcal{E}$ as

$$
\begin{aligned}
\ln \left[\lambda^{2 n}\right] & =2 n \ln [\lambda]=2 \ln [\lambda] \frac{\ln \mathcal{E}^{-1}+\ln \left[2\left(p_{\max }\right)^{-1}\right]}{\ln Q^{-1}} \\
& =\ln \mathcal{E}^{-1} \frac{2 \ln [\lambda]}{\ln Q^{-1}}+\frac{\ln [\lambda] \ln \left[4\left(p_{\max }\right)^{-2}\right]}{\ln Q^{-1}},
\end{aligned}
$$

and it follows that

$$
\begin{aligned}
& \lambda^{2 n}=\exp \left[\ln \mathcal{E}^{-1} \frac{2 \ln [\lambda]}{\ln Q^{-1}}+\frac{\ln [\lambda] \ln \left[4\left(p_{\max }\right)^{-2}\right]}{\ln Q^{-1}}\right] \\
& =\mathcal{E}^{2 \ln \left[\lambda^{-1}\right] / \ln Q^{-1}} \exp \left[\frac{\ln [\lambda] \ln \left[4\left(p_{\max }\right)^{-2}\right]}{\ln Q^{-1}}\right] .
\end{aligned}
$$

We can finally express the number of samples explicitly as

$$
\begin{aligned}
N_{s}= & 4 \operatorname{prob}_{0}\left(1-\operatorname{prob}_{0}\right) \mathcal{E}^{-2\left\{1+\left[\ln \left(\lambda^{-1}\right) / \ln \left(Q^{-1}\right)\right]\right\}} \\
& \times \exp \left[\frac{\ln \left(\lambda^{-1}\right) \ln \left[4\left(p_{\max }\right)^{-2}\right]}{\ln \left(Q^{-1}\right)}\right] \\
= & \mathcal{O}\left[\mathcal{E}^{-2(1+f)}\right] .
\end{aligned}
$$

Here we used that $4 \exp \left\{\ln \left[\lambda^{-1}\right] \ln \left[4\left(p_{\max }\right)^{-2}\right] / \ln \left(Q^{-1}\right)\right\}$ is a constant multiplication factor and $0 \leq \operatorname{prob}_{0} \leq 1$ and we have introduced $f:=\ln \left(\lambda^{-1}\right) / \ln \left(Q^{-1}\right)$. Indeed, we obtain the expected limits due to $\lim _{\lambda \rightarrow 1} f=0$ and $\lim _{Q \rightarrow 0} f=0$.

In general when $\lambda>1 / 2$ we can use the expression $Q \leq$ $\left(\lambda^{-1}-1\right)$ from Theorem 2 as $f \leq \ln \left(\lambda^{-1}\right) / \ln \left[\left(\lambda^{-1}-1\right)^{-1}\right]$, which is only saturated by 0 -entropy distributions. For example, when $\lambda=0.6$, then we obtain $f \leq 1.26$, and this value can be smaller depending on the entropy 
of the probability distribution. Interestingly, for sufficiently good quality states as $\lambda \geq 0.9$, the polynomial overhead introduced is very small via $f \leq 0.16$.

Method A: In this case we estimate both $\operatorname{prob}_{0}$ and $\operatorname{prob}_{0}^{\prime}$. The variance of our estimator can be specified as

$$
\begin{aligned}
\mathcal{E}^{2} & =\operatorname{Var}[\langle\psi|\sigma| \psi\rangle]=\operatorname{Var}\left[\frac{2 \operatorname{prob}_{0}-1}{2 \operatorname{prob}_{0}^{\prime}-1}\right] \\
& =\operatorname{Var}\left[\operatorname{prob}_{0}\right] \frac{4}{\left(2 \operatorname{prob}_{0}^{\prime}-1\right)^{2}}+\operatorname{Var}\left[\operatorname{prob}_{0}^{\prime}\right] \frac{4\left(2 \operatorname{prob}_{0}-1\right)^{2}}{\left(2 \operatorname{prob}_{0}^{\prime}-1\right)^{4}}
\end{aligned}
$$

Let us now use that $2 \operatorname{prob}_{0}^{\prime}-1 \approx \lambda^{n}$ and simplify the above expression as

$$
\begin{aligned}
\mathcal{E}^{2} & =\operatorname{Var}[\langle\psi|\sigma| \psi\rangle] \approx \operatorname{Var}\left[\frac{2 \operatorname{prob}_{0}-1}{2 \operatorname{prob}_{0}^{\prime}-1}\right] \\
& =\operatorname{Var}\left[\operatorname{prob}_{0}\right] \frac{4}{\lambda^{2 n}}+\operatorname{Var}\left[\operatorname{prob}_{0}^{\prime}\right] \frac{4\left(2 \operatorname{prob}_{0}-1\right)^{2}}{\lambda^{4 n}} .
\end{aligned}
$$

We can again substitute the variance of binomial distributions as $\operatorname{Var}\left[\operatorname{prob}_{0}\right]=\operatorname{prob}_{0}\left(1-\operatorname{prob}_{0}\right) / N_{s, 1}$ and $\operatorname{Var}\left[\operatorname{prob}_{0}^{\prime}\right]=\operatorname{prob}_{0}^{\prime}\left(1-\operatorname{prob}_{0}^{\prime}\right) / N_{s, 2}$. The measurement cost of determining both components to a precision $\mathcal{E}^{2} / 2$ follows as

$$
\begin{aligned}
& N_{s, 1}=\frac{8 \operatorname{prob}_{0}\left(1-\operatorname{prob}_{0}\right)}{\mathcal{E}^{2} \lambda^{2 n}} \text { and } \\
& N_{s, 2}=\frac{8 \operatorname{prob}_{0}^{\prime}\left(1-\operatorname{prob}_{0}^{\prime}\right)\left(2 \operatorname{prob}_{0}-1\right)^{2}}{\mathcal{E}^{2} \lambda^{4 n}} .
\end{aligned}
$$

We can now use our previous expression from Eq. (B13) for determining $\lambda^{2 n}$ and $\lambda^{4 n}$, which finally yields our formula for the measurement costs as

$$
N_{s, 1}=\mathcal{O}\left[\mathcal{E}^{-2(1+f)}\right] \quad \text { and } \quad N_{s, 2}=\mathcal{O}\left[\mathcal{E}^{-2(1+2 f)}\right] .
$$

The total number of measurements required to determine the result is indeed $N_{s, 1}+N_{s, 2}$, and recall that $f=$ $\ln \left(\lambda^{-1}\right) / \ln \left(Q^{-1}\right)$.

\section{APPENDIX C: EFFECT OF VIOLATING ASSUMPTIONS}

Let us now analyze how nonidentical copies of $\rho$ affect the performance of our approach.

Lemma 3.-When the states are not perfectly identical via $\rho=\otimes_{\mu=1}^{n} \rho_{\mu}$ with $\rho_{1} \neq \rho_{2} \cdots \neq \rho_{n}$, but their largest eigenvector is identical, then our main result from Theorem 2 still holds and we still obtain exponentially decreasing error bounds as
Method A: $\quad \frac{2 \operatorname{prob}_{0}-1}{2 \operatorname{prob}_{0}^{\prime}-1}=\langle\psi|\sigma| \psi\rangle+\mathcal{O}\left(\left[\lambda_{\text {min }}^{-1}-1\right]^{n}\right)$,

Method B : $\quad \frac{2 \operatorname{prob}_{0}-1}{\prod_{\mu=1}^{n} \lambda_{\mu}}=\langle\psi|\sigma| \psi\rangle+\mathcal{O}\left(\left[\lambda_{\text {min }}^{-1}-1\right]^{n}\right)$.

For method B we assume that the dominant eigenvalues $\lambda_{1}, \lambda_{2}, \ldots, \lambda_{n}$ are known. The error depends on the smallest of these dominant eigenvalues, which we denote as $\lambda_{\min }$. In the special case when all $\rho_{\mu}$ commute (i.e., same eigenvectors, but different eigenvalues) our error bounds $\mathcal{E}_{A}$ and $\mathcal{E}_{B}$ from Theorem 2 approximately hold via an effective sequence $Q_{n}^{\text {eff }}$, and we can expect an error suppression very similar to Theorem 2.

Proof-Case 1: Let us build up components of our proof by first considering the special case when all $\rho_{k}$ commute with each other. In other words, the states satisfy the spectral decomposition

$$
\rho_{\mu}=\lambda_{\mu}|\psi\rangle\left\langle\psi\left|+\left(1-\lambda_{\mu}\right) \sum_{k=2}^{d} p_{k_{\mu}}\right| \psi_{k}\right\rangle\left\langle\psi_{k}\right|,
$$

and they all share the same eigenvectors while their eigenvalues can be different. It follows that the orthogonality relations in the proof of Theorem 1 in Eq. (A4) still hold and the final result can be written explicitly as

$$
\begin{aligned}
\operatorname{prob}_{0}= & \frac{1}{2}+\frac{1}{2}\langle\psi|\sigma| \psi\rangle \prod_{\mu=1}^{n} \lambda_{\mu} \\
& +\sum_{k=2}^{d}\left\langle\psi_{k}|\sigma| \psi_{k}\right\rangle \prod_{\mu=1}^{n} p_{k_{\mu}}\left(1-\lambda_{\mu}\right) .
\end{aligned}
$$

We can upper bound the error term in the above expression as

$$
\begin{gathered}
\left|\sum_{k=2}^{d}\left\langle\psi_{k}|\sigma| \psi_{k}\right\rangle \prod_{\mu=1}^{n} p_{k_{\mu}}\left(1-\lambda_{\mu}\right)\right| \\
\leq\left(1-\lambda_{\min }\right)^{n} \sum_{k=2}^{d}\left(p_{k, \max }\right)^{n}
\end{gathered}
$$

where we have denoted the largest component as $\lambda_{\min }=$ $\min _{\mu} \lambda_{\mu}$ and $p_{k, \max }=\max _{\mu} p_{k_{\mu}}$ and we have also used that $\left|\left\langle\psi_{k}|\sigma| \psi_{k}\right\rangle\right| \leq 1$.

We can also upper bound the product $\prod_{\mu=1}^{n} \lambda_{\mu} \geq \lambda_{\min }^{n}$ and derive the error of our method $\mathrm{A}$ in Theorem 2, which results in

$$
\frac{2 \operatorname{prob}_{0}-1}{2 \operatorname{prob}_{0}^{\prime}-1}=\langle\psi|\sigma| \psi\rangle+\mathcal{E}_{A}, \quad \text { with } \quad\left|\mathcal{E}_{A}\right| \leq \frac{2 Q_{n}^{\text {eff }}}{1+Q_{n}^{\text {eff }}},
$$

which we write in terms of an effective sequence $Q_{n}^{\mathrm{eff}}=\left(\lambda_{\min }^{-1}-1\right)^{n}\left\|\underline{p}_{\max }\right\|_{n}^{n}$. 
We can similarly derive the errors of our method B in Theorem 2 in the case when the eigenvalues $\lambda_{1}, \lambda_{2}, \ldots, \lambda_{n}$ are known. This results in

$$
\frac{2 \operatorname{prob}_{0}-1}{\prod_{\mu=1}^{n} \lambda_{\mu}}=\langle\psi|\sigma| \psi\rangle+\mathcal{E}_{B}, \quad \text { with } \quad\left|\mathcal{E}_{B}\right| \leq Q_{n}^{\text {eff }},
$$

where we have again used our effective sequence $Q_{n}^{\text {eff }}=\left(\lambda_{\min }^{-1}-1\right)^{n}\left\|\underline{p}_{\max }\right\|_{n}^{n}$. We note that here $\underline{p}_{\max }$ is no longer a proper probability vector since $\sum_{k=2}^{d} p_{k, \max } \geq 1$ and therefore we cannot guarantee in general that the resulting suppression factor $Q_{\text {eff }}<1$. Nevertheless, one expects a very similar exponential decay of the error as in Theorem 2 and in Lemma 1 for high-entropy probability distributions and for $n>1$.

Case 2: We now consider the most general case when $\rho_{\mu}$ are arbitrary except that their dominant eigenvector is exactly $|\psi\rangle$. The states therefore admit the following spectral decomposition:

$$
\rho_{\mu}=\lambda_{\mu}|\psi\rangle\left\langle\psi\left|+\left(1-\lambda_{\mu}\right) \sum_{k=2}^{d} p_{k_{\mu}}\right| \psi_{k_{\mu}}\right\rangle\left\langle\psi_{k_{\mu}}\right|,
$$

It follows from the above definition that the dominant eigenvector $|\psi\rangle$ is orthogonal to every error contribution in every eigenstate as $\left\langle\psi \mid \psi_{k_{\mu}}\right\rangle=0$ for every $k=\left\{2, \ldots, 2^{N}\right\}$ and for every $\mu=\{1, \ldots, n\}$. Modifying accordingly the orthogonality relation in the proof of Theorem 1 in Eq. (A4) allows us to compute the leading term as expected, but every other nonzero term is multiplied with the prefactor $\prod_{\mu=1}^{n}\left(1-\lambda_{\mu}\right)$ which leads to the following error term:

$$
\operatorname{prob}_{0}=\frac{1}{2}+\frac{1}{2}\langle\psi|\sigma| \psi\rangle \prod_{\mu=1}^{n} \lambda_{\mu}+\mathcal{O}\left[\prod_{\mu=1}^{n}\left(1-\lambda_{\mu}\right)\right] .
$$

As shown previously, this allows us to compute the error of our method A and method B in Theorem 2 as

$$
\begin{aligned}
& \frac{2 \operatorname{prob}_{0}-1}{2 \operatorname{prob}_{0}^{\prime}-1}=\langle\psi|\sigma| \psi\rangle+\mathcal{O}\left(\left[\lambda_{\text {min }}^{-1}-1\right]^{n}\right) \quad \text { and } \\
& \frac{2 \operatorname{prob}_{0}-1}{\prod_{\mu=1}^{n} \lambda_{\mu}}=\langle\psi|\sigma| \psi\rangle+\mathcal{O}\left(\left[\lambda_{\text {min }}^{-1}-1\right]^{n}\right),
\end{aligned}
$$

in general for $\lambda_{\min }>1 / 2$.

\section{Coherent mismatch in incoherent error channels}

As we discussed in the main text, our approach cannot address coherent errors, i.e., when the dominant eigenvector of the density matrix is $\sqrt{1-c}\left|\psi_{\text {id }}\right\rangle+\sqrt{c}\left|\psi_{\text {err }}\right\rangle$, where $\psi_{\text {id }}$ is the ideal computational state and $\psi_{\text {err }}$ is some error. This is expected to happen when systematic errors, such as miscalibrated rotation angles, are present, but it is straightforward to show that even a completely incoherent error channel (random unitary events) can introduce a slight mismatch in the eigenvectors.

We show this by considering a quite general noise channel as

$$
\rho^{\prime}=(1-\epsilon) \rho+\epsilon \rho_{\mathrm{err}},
$$

in which no errors happen with a probability $(1-\epsilon)$ and some error happens with a probability $\epsilon$. In complete generality, the eigenvectors of $\rho$ can be different than the eigenvectors of $\rho_{\text {err }}$ unless the commutator vanishes $\left[\rho, \rho_{\text {err }}\right]=0$. A typical example for a vanishing commutator is the single-qubit depolarizing channel in single-qubit systems, in which case $\rho^{\prime}=(1-\epsilon) \rho+\epsilon$ Id and indeed $[\rho, \mathrm{Id}]=0$. However, for more than 1 qubit (or nonseparable states) the above expression does not hold and even single-qubit depolarizing can introduce a coherent mismatch such that the dominant eigenvector of $\rho^{\prime}$ is $\sqrt{1-c}|\psi\rangle+\sqrt{c}\left|\psi_{\mathrm{err}}\right\rangle$.

The coherent mismatch due to incoherent errors is expected to be very small in practically relevant scenarios since the high entropy of the error probabilities from Eq. (1) ensures us that $\left\|\left[\rho, \rho_{\mathrm{err}}\right]\right\| \ll 1$. For example, in our numerical simulations in Fig. 2 the infidelity of the dominant eigenvector with respect to the pure state obtained from a noise-free computation was below $10^{-4}$.

In general, for a high-entropy error distribution in Eq. (1) we obtain the spectral decomposition with $\lambda_{k} \ll 1$ for $k \geq 2$ :

$$
\rho=\sum_{k=1} \lambda_{k}\left|\psi_{k}\right\rangle\left\langle\psi_{k}\right|
$$

One can compute the first-order (in $\epsilon$ ) corrections to the eigenvectors of $\rho^{\prime}$ via the usual perturbative series: the dominant eigenvector of $\rho^{\prime}$ is approximately (up to normalization)

$$
\begin{aligned}
\left|\psi_{1}^{\prime}\right\rangle & \approx\left|\psi_{1}\right\rangle+\sum_{k=2} \frac{\left\langle\psi_{k}\left|\epsilon \rho_{\mathrm{err}}\right| \psi_{1}\right\rangle}{\lambda_{1}-\lambda_{k}}\left|\psi_{k}\right\rangle \\
& =\left|\psi_{1}\right\rangle+\frac{\epsilon}{\lambda_{1}} \sum_{k=2}\left\langle\psi_{k}\left|\rho_{\mathrm{err}}\right| \psi_{1}\right\rangle\left|\psi_{k}\right\rangle=\left|\psi_{1}\right\rangle+\mathcal{O}(\epsilon),
\end{aligned}
$$

where we have used that $\lambda_{1}-\lambda_{k} \approx \lambda_{1}$. Indeed the result is constant bounded due to the norm of the fist-order correction $\sum_{k=2}\left|\left\langle\psi_{k}\left|\rho_{\text {err }}\right| \psi_{1}\right\rangle\right|^{2}=\left|\operatorname{Col}_{1}\left[\rho_{\text {err }}\right]\right|^{2} \leq 1$, hence the scaling of the correction $\mathcal{O}(\epsilon)$. Here $\operatorname{Col}_{1}\left[\rho_{\text {err }}\right]$ is the column vector of $\rho_{\text {err }}$ whose norm is bounded by the largest eigenvalue.

Let us now focus on the repeated application of the noise channel from Eq. (C4) which can be used to model a quantum circuit that applies a series of noisy quantum gates with error probability $\epsilon$. The incoherent decay of the dominant eigenvalue is expected to decay exponentially with $\nu$ as $(1-\epsilon)^{\nu}$. For large systems one needs to 
implement a large number $\nu$ of gates and therefore one requires to have a sufficiently low per-gate error $\epsilon$ in order to keep the dominant eigenvalue above a threshold $\lambda_{\min }-$ and thus keep the sampling costs in result 2 practical. Since the strength of the coherent mismatch is proportional to the per-gate error rate $\epsilon$, it is expected to decrease when we decrease $\epsilon$. Interestingly, our numerical simulations of the single- and 2-qubit depolarizing channel suggest that in the investigated region (see Fig. 5) the coherent mismatch only grows linearly when we increase the number of gates $\nu$. This suggest that when we increase the number of gates the incoherent (exponential) decay of the dominant eigenvalue is significantly more damaging than the (linearly) increasing coherent mismatch.

We illustrate this the following way. Let us define the following quantities. We define the fidelity between the dominant eigenvector $\psi_{1}^{(\nu)}$ (after the application of $\nu$ noisy gates) and the ideal state as $\eta_{1}:=\left|\left\langle\psi_{\text {id }} \mid \psi_{1}^{(\nu)}\right\rangle\right|^{2}=1-c$. Furthermore, we define the fidelity between the dominant eigenvector and the density matrix $\rho$ as $\eta_{2}:=$ $\left\langle\psi_{1}^{(\nu)}|\rho| \psi_{1}^{(\nu)}\right\rangle \approx \lambda$. Here $\eta_{1}$ decays due to the coherent mismatch while $\eta_{2} \approx(1-\epsilon)^{\nu}$ decays purely due to the incoherent effect of the noise channel. Figure 6(a) shows how the ratio $\eta_{2} / \eta_{1}$ decreases when we increase the number of gates. Interestingly the scale at which this ratio decays appears to be exponential in the investigated region.

These results suggest that the coherent mismatch in the dominant eigenvector can be expected to be sufficiently small for large, complex quantum circuits. Refer to Ref. [45] for a detailed analysis.

\section{a. Mitigating the coherent mismatch}

As discussed in the main text, well-established techniques can be used to mitigate the effect of coherent errors.
We now focus on the above introduced coherent mismatch in the eigenvector due to incoherent error channels and demonstrate the effectiveness of an extrapolation approach in Fig. 6(b). Similarly to Fig. 3 in the main text, we use extrapolation techniques, but here we vary the gate error rate in the state preparation stage (and not in the derangement process). We set gate errors such that 2-qubit gates undergo a depolarizing noise with probability $\epsilon=10^{-3}$ and assume that the experimentalist can increase this error in $k=2,3,4, \ldots$ steps up to $\epsilon=10^{-2}$. As expected from the above arguments based on a perturbative expansion of the dominant eigenvector, the measured expectation value should depend on the error levels as a polynomial that has rapidly decaying expansion coefficients due to the fact that the per-gate error level is low as $\epsilon \ll 1$. We have determined extrapolation errors using various fitting techniques as shown in Fig. 6(b). We define the extrapolation error as the difference between the ideal, error-free expectation value $\left\langle\psi_{\text {id }}|\sigma| \psi_{\text {id }}\right\rangle$ and the estimated expected value $\operatorname{Tr}\left[\rho^{n} \sigma\right] / \operatorname{Tr}\left[\rho^{n}\right]$ from method A of Theorem 2. Here $\left|\psi_{\text {id }}\right\rangle$ is the state that one would obtain from a perfect, noise-free evaluation of the circuit and in our simulation we consider the same 12-qubit circuit as in Fig. 2 (right) in the main text (refer to Appendix F) with $n=3$ copies.

Indeed, Fig. 6(b) confirms that the effect of the coherent mismatch can be straightforwardly mitigated by fitting loworder polynomials to the experimental data. The red horizontal line represents the error bound from result 1 and one can demonstrably suppress the effect of the coherent mismatch below this error bound. As expected, when we increase the degree of the fitting polynomial, the error saturates as it reaches the level from result 1 , which we defined for the case when the coherent mismatch is neglected-and the errors could only be further suppressed by increasing the number $n$ of copies. (a)

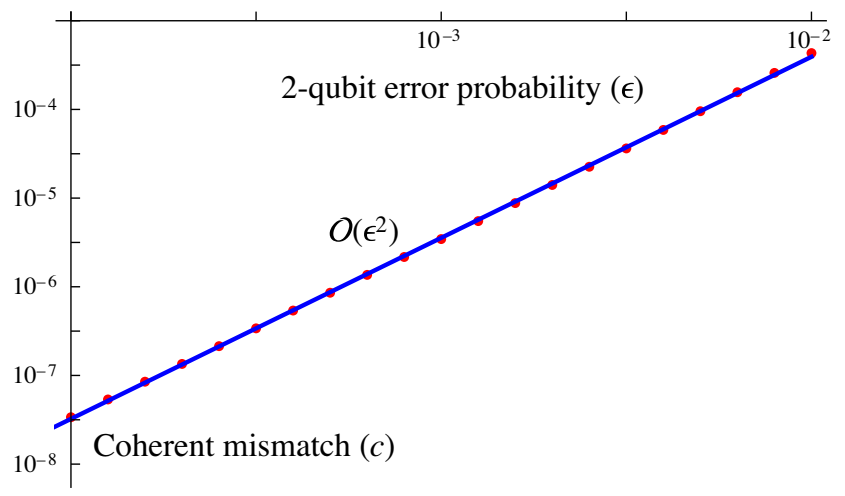

(b)

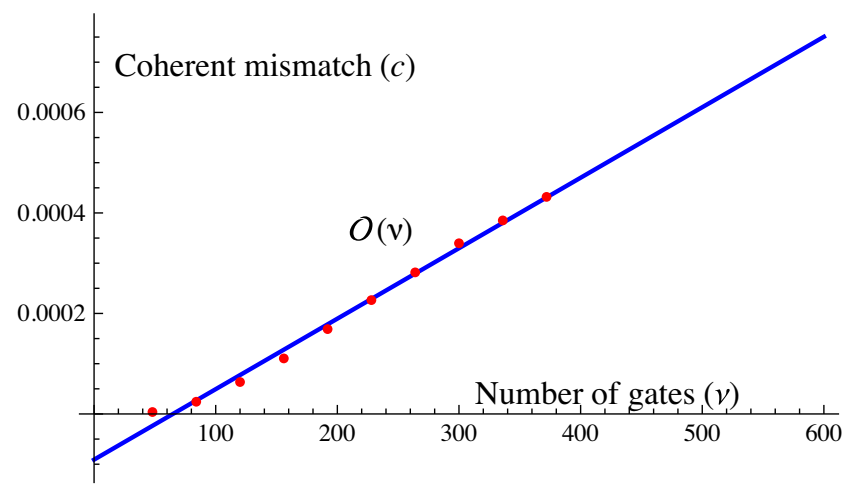

FIG. 5. Coherent mismatch $c$ in the dominant eigenvector $\sqrt{1-c}|\psi\rangle+\sqrt{c}\left|\psi_{\text {err }}\right\rangle$ of the density matrix $\rho$ in case of a purely incoherent noise model (single- and 2-qubit depolarizing). We simulated the same 12-qubit system with 372 noisy gates from Fig. 2 in which single-qubit gates undergo single-qubit depolarization with probability $0.1 \epsilon$ while 2-qubit gates undergo 2-qubit depolarization with probability $\epsilon$. (a) The coherent mismatch is small and scales with the per-gate error $\epsilon$ as $c=\mathcal{O}\left(\epsilon^{2}\right)$ as explained in the text. (b) The coherent mismatch is small and it grows with the number of gates $\nu$ at a fixed gate error $\epsilon=10^{-3}$ as $c=\mathcal{O}(\nu)$. 


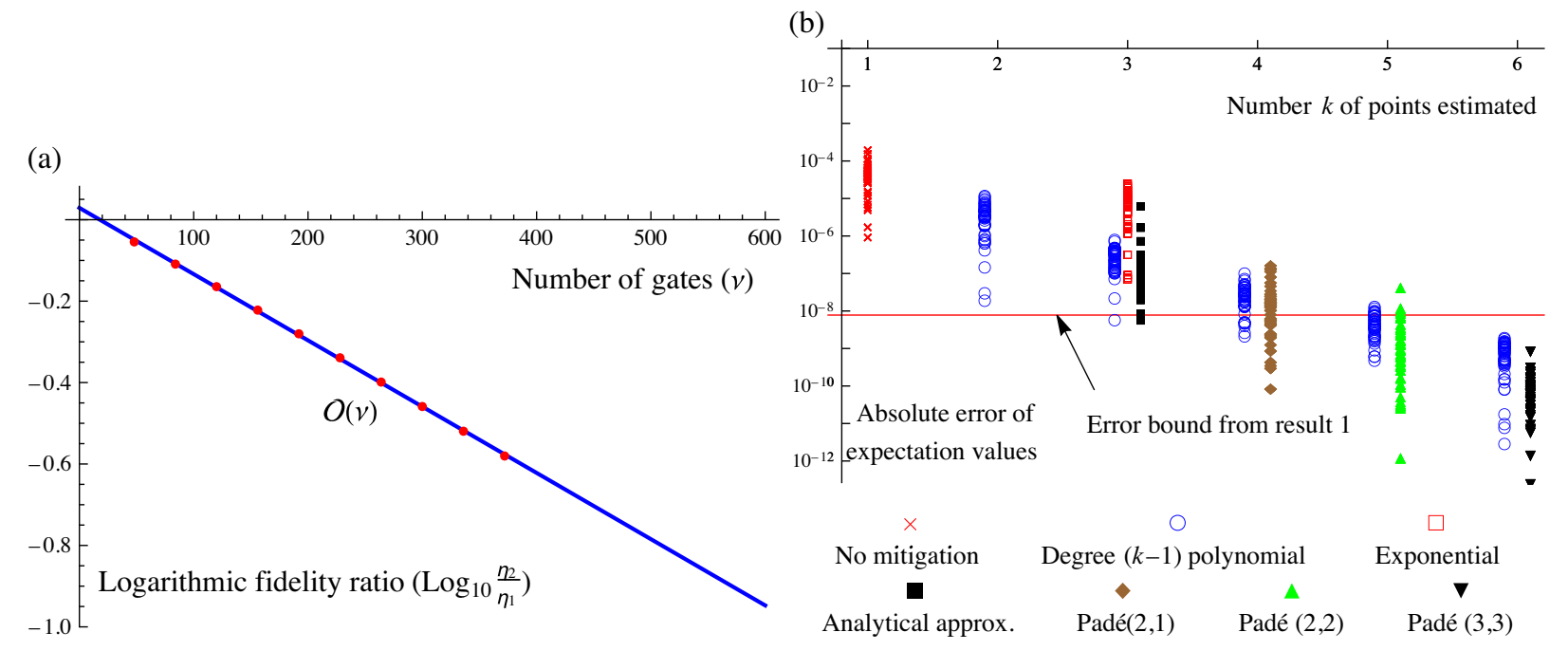

FIG. 6. (a) The fidelity $\eta_{1}$ decreases purely due to the coherent mismatch while the fidelity $\eta_{2}$ decays purely due to the incoherent effect of the noise channel as discussed in Appendix $\mathrm{C} 1$. The ratio $\eta_{2} / \eta_{1}$ appears to decay exponentially in the investigated region (linear in the logarithmic plot) when we increase the number of gates for the fixed 2-qubit gate error $\epsilon=10^{-2}$ and this ensures us that the coherent mismatch in the dominant eigenvector becomes negligible for large systems (large $\nu$ ). We simulated the same circuit as in Fig. 5(b). (b) Mitigating the error caused by a coherent mismatch in the dominant eigenvector of the density matrix as discussed in Appendix C 1. Gate error levels in the state-preparation stage were varied in $k=2,3,4, \ldots$ steps and the obtained expectation values were extrapolated to the zero error limit. The red horizontal line represents the error bound from result 1 , and one can straightforwardly suppress the effect of the coherent mismatch below this error bound by fitting low-order polynomials. The error saturates when increasing the degree of the fitting polynomial and can only be further reduced by increasing the number $n$ of copies.

\section{APPENDIX D: NOISE RESILIENCE OF DERANGEMENTS AND ERROR EXTRAPOLATION}

Example 3: We now show examples why the derangement operator is highly resilient to errors. We proceed by recapitulating that quantum channels can be represented by a set of nonunique Kraus maps and, in particular, we consider the decomposition into the following sum of unitary transformations as

$$
\rho^{\prime}=(1-\epsilon) U \rho U^{\dagger}+\epsilon \sum_{m} c_{m} U_{m} \rho U_{m}^{\dagger},
$$

where $U$ is the ideal unitary transformation, $\sum_{k} c_{m}=1$ and $0 \leq \epsilon, c_{m} \leq 1$, while the erroneous Kraus operators are unitary via $U_{m} U_{m}^{\dagger}=$ Id. The deviation from the ideal transformation can be interpreted as unitary transformations that randomly affect the eigenvectors $|\psi\rangle$ of the quantum state as $U_{m}|\psi\rangle$ with probability $\epsilon c_{m}$.

Let us now analyze how such errors affect our procedure when they occur during the derangement operator; i.e., we set the ideal transformation $U$ to be our derangement circuit from Fig. 1. First, we show that the orthogonality relations in the proof of Theorem 1 are resilient to such noise events. In particular, recall that the derangement operator symmetrizes the input state as, e.g.,

$$
D_{n}\left|\psi_{1}, \psi_{2}, \ldots, \psi_{n}\right\rangle=\left|\psi_{n}, \psi_{1}, \ldots, \psi_{n-1}\right\rangle \text {, }
$$

which would ideally ensure that only permutationsymmetric combinations contribute to the output via the orthogonality relation from Eq. (A4) as

$$
\begin{aligned}
& \left\langle\psi_{k_{1}}, \psi_{k_{2}}, \ldots, \psi_{k_{n}} \mid \sigma \psi_{k_{n}}, \psi_{k_{1}}, \ldots, \psi_{k_{n-1}}\right\rangle \\
& \quad=\left\langle\psi_{k_{1}} \mid \sigma \psi_{k_{n}}\right\rangle\left\langle\psi_{k_{2}} \mid \psi_{k_{1}}\right\rangle \cdots\left\langle\psi_{k_{n}} \mid \psi_{k_{n-1}}\right\rangle .
\end{aligned}
$$

One can show that even if errors occur during the derangement procedure, the orthogonality relations are still preserved as

$$
\begin{aligned}
& \left\langle U_{1} \psi_{k_{1}}, U_{2} \psi_{k_{2}}, \ldots, U_{n} \psi_{k_{n}} \mid \sigma U_{1} \psi_{k_{n}}, U_{2} \psi_{k_{1}}, \ldots, U_{n} \psi_{k_{n-1}}\right\rangle \\
& =\left\langle U_{1} \psi_{k_{1}} \mid \sigma U_{1} \psi_{k_{n}}\right\rangle\left\langle\psi_{k_{2}} \mid \psi_{k_{1}}\right\rangle \cdots\left\langle\psi_{k_{n}} \mid \psi_{k_{n-1}}\right\rangle .
\end{aligned}
$$

It follows that the nonsymmetric combinations of input states do not contribute to the output even when the derangement operator is affected by random errors. Note that even though the errors do not directly contribute to the final output (as shown above), the probability that the circuit outputs an error-free result is decreased via the $1-\epsilon$ factor. This is, however, a trivial effect that only attenuates the output probabilities linearly and can be completely corrected by a linear extrapolation (i.e., estimating the output probabilities at different $\epsilon$ values and then extrapolating to $\epsilon=0$ ).

Second, let us show that for symmetric input states $|\psi, \psi, \ldots, \psi\rangle$ all random errors during the derangement procedure cancel that do not affect the ancilla qubit nor the 
register to which the observable $\sigma$ is applied. In fact, we just modify the above equation by not allowing errors on register 1 as

$$
\begin{aligned}
& \left\langle\psi, U_{2} \psi, \ldots, U_{n} \psi \mid \sigma \psi, U_{2} \psi, \ldots, U_{n} \psi\right\rangle \\
& \quad=\langle\psi \mid \sigma \psi\rangle\langle\psi \mid \psi\rangle \cdots\langle\psi \mid \psi\rangle=\langle\psi|\sigma| \psi\rangle .
\end{aligned}
$$

The second equation shows that we obtain the correct contribution despite all registers except for register 1 have undergone some random error $U_{2}, U_{3}$, etc. Our previous argument again holds: despite the fact that these error events do not directly contribute to the final output of the circuit, the probability of an error-free output is attenuated linearly which, nonetheless, can be completely corrected by a linear extrapolation.

In summary, the derangement measurement is highly resilient to errors and completely protects the permutation symmetry of input states even when the derangement operator suffers from experimental noise. However, errors that affect the qubits to which the observable $\sigma$ is applied will degrade the final result nontrivially via $\left\langle U_{1} \psi|\sigma| U_{1} \psi\right\rangle$, where $U_{1}$ is some unitary noise process that occurs with a (possibly) low probability. Nevertheless, we show in the main text and in the following theorem that these erroneous contributions can be successfully mitigated with, e.g., extrapolation techniques.

Theorem 3.-Assume that a circuit consists of a sequence of $\nu$ noisy quantum gates, and each gate's error model is of the form $(1-\epsilon) \Phi_{k}+\epsilon \mathcal{E}_{k}$, where $\Phi_{k}$ is the ideal, error-free quantum channel and $\mathcal{E}_{k}$ is an arbitrary error channel (completely positive trace preserving map) that occurs with probability $\epsilon$. Most typical error models are of this form, including dephasing, depolarizing, inhomogeneous Pauli errors, arbitrary unital channels, and beyond ( $\mathcal{E}_{k}$ need not be local or two-local). In a circuit that consists of number $\nu$ such gates, any expectation value $E$ will depend on the error probability $\epsilon$ as a degree $\nu$ polynomial via

$$
E(\epsilon)=E_{0}+\sum_{k=1}^{\nu} \epsilon^{k} E_{k}
$$

where $E_{k}$ are real polynomial coefficients. One can therefore exactly determine the ideal expectation value $E(0)$ by estimating $E(\epsilon)$ at $\nu+1$ points in $\epsilon$. The so-called Lagrange polynomial or the Newton polynomial provide explicit formulas for computing $E(\epsilon)$ from the pointwise reconstructions $E\left(\epsilon_{k}\right)$. Furthermore, one can approximate the dependence on $\epsilon$ via, e.g., the $(3,3)$ Padé approximation as

$$
E(\epsilon) \approx E_{0}-\tilde{\eta} \epsilon \frac{(1-\epsilon)^{\nu}}{2 \epsilon-1} \approx \frac{a_{1} \epsilon+a_{2} \epsilon^{2}+a_{3} \epsilon^{3}}{1+a_{4} \epsilon+a_{5} \epsilon^{2}}
$$

that only requires the coefficients $a_{1}, a_{2}, a_{3}, a_{4}, a_{5}$ to be fitted to experimental data.

Proof.-Applying $\nu$ gates in a sequence will result in the product of channels,

$$
\prod_{k=1}^{\nu}\left[(1-\epsilon) \Phi_{k}+\epsilon \mathcal{E}_{k}\right]=(1-\epsilon)^{\nu} \prod_{k=1}^{\nu} \Phi_{k}+\sum_{k=1}^{\nu}(1-\epsilon)^{\nu-k} \epsilon^{k} \mathcal{G}_{k},
$$

where $\mathcal{G}_{k}$ is a channel which decomposes into the sum of all terms in which $k$ errors occur and $\prod_{k=1}^{\nu} \Phi_{k}$ is the ideal error-free circuit. We can introduce the circuit with no errors as $\mathcal{G}_{0}:=\prod_{k=1}^{\nu} \Phi_{k}$, which simplifies our formula as

$$
\prod_{k=1}^{\nu}\left[(1-\epsilon) \Phi_{k}+\epsilon \mathcal{E}_{k}\right]=\sum_{k=0}^{\nu}(1-\epsilon)^{\nu-k} \epsilon^{k} \mathcal{G}_{k}
$$

It follows that any expectation value (with respect to some observable $\mathcal{H}$ ) will be of the form

$$
\begin{aligned}
E & =\operatorname{Tr}\left\{\mathcal{H} \prod_{k=1}^{\nu}\left[(1-\epsilon) \Phi_{k}+\epsilon \mathcal{E}_{k}\right] \rho\right\} \\
& =\sum_{k=0}^{\nu}(1-\epsilon)^{\nu-k} \epsilon^{k} \operatorname{Tr}\left\{\mathcal{H} \mathcal{G}_{k} \rho\right\}
\end{aligned}
$$

therefore, any expectation value can be expressed as a degree $\nu$ polynomial as a function of the error probability as

$$
E(\epsilon)=E_{0}+\sum_{k=1}^{\nu} \epsilon^{k} E_{k},
$$

where $E_{0}$ is the ideal, noise-free expectation value and $E_{k}$ are polynomial coefficients.

Let us now write (without loss of generality) that the expectation values are of the form $\operatorname{Tr}\left\{\mathcal{H} \mathcal{G}_{k} \rho\right\}=\tilde{\eta}+\eta_{k}$, where $\tilde{\eta}$ is a mean value and $\eta_{k}$ expresses the deviation from the mean value. Let us assume that $\eta_{k} \ll \tilde{\eta}$, which in the case of the derangement operator is motivated by our argument in example 3 , that most errors do not contribute and therefore $\tilde{\eta} \approx 0$. In this case we can evaluate the summation analytically for the mean value:

$$
\begin{aligned}
E(\epsilon) & =E_{0}+\sum_{k=1}^{\nu}(1-\epsilon)^{\nu-k} \epsilon^{k} \operatorname{Tr}\left\{\mathcal{H} \mathcal{G}_{k} \rho\right\} \\
& =E_{0}+\tilde{\eta} \sum_{k=0}^{\nu}(1-\epsilon)^{\nu-k} \epsilon^{k}+\mathcal{O}\left(\eta_{k}\right) \\
& =E_{0}+\tilde{\eta} \epsilon \frac{\epsilon^{\nu}-(1-\epsilon)^{\nu}}{2 \epsilon-1}+\mathcal{O}\left(\eta_{k}\right) .
\end{aligned}
$$

We can obtain a Padé expansion of the above result at $\epsilon \approx 0$ by neglecting the term $\epsilon^{\nu}$. For example, the $(3,3)$ Padé approximation follows as 


$$
\begin{aligned}
E(\epsilon) & \approx E_{0}-\tilde{\eta} \epsilon \frac{(1-\epsilon)^{\nu}}{2 \epsilon-1} \\
& \approx E_{0}-\tilde{\eta} \frac{\epsilon+a(n) \epsilon^{2}+b(n) \epsilon^{3}}{1+c(n) \epsilon+d(n) \epsilon^{2}},
\end{aligned}
$$

where $a(n), b(n), c(n), d(n)$ are the Padé expansion coefficients that depend on the number $n$ of gates $\nu$, for example,

$$
a(n)=\frac{2\left(62+11 n-8 n^{2}+n^{3}\right)}{5\left(26-9 n+n^{2}\right)} .
$$

Indeed this expansion is only valid when $\eta_{k} \approx 0$. Nevertheless, we propose to approximate the polynomial

$$
E(\epsilon) \approx \frac{a_{1} \epsilon+a_{2} \epsilon^{2}+a_{3} \epsilon^{3}}{1+a_{4} \epsilon+a_{5} \epsilon^{2}},
$$

by fitting the coefficients $a_{1}, a_{2}, a_{3}, a_{4}, a_{5}$ to experimental data.

\section{APPENDIX E: HARDWARE-NATIVE IMPLEMENTATION OF DERANGEMENT CIRCUITS}

\section{Recompiling controlled-sWAP gates}

Recall that derangement circuits permute registers via Definition 1. Permuting two registers is performed by the SWAP operator, which decomposes into a product of $N$ elementary, 2-qubit SWAP gates as $\operatorname{SWAP}_{N, N^{\prime}} \cdots \mathrm{SWAP}_{2,2^{\prime}} \mathrm{SWAP}_{1,1^{\prime}}$, where $N$ is the number of qubits in a register. Our aim is now to optimally recompile elementary, controlled-sWAP gates assuming various different hardware-native gate sets.

The controlled-SWAP, also called Fredkin, gate has been much investigated in the literature, but mostly in the context of fault-tolerant quantum computing. For example, Ref. [101] provided a circuit that optimally implements the controlled-SWAP gate using 8 applications of CNOT gates and 9 applications of $T$ gates. Early works have suggested that if one has the ability to natively implement any 2-qubit gate, then one can in principle implement the controlledSWAP gate with only 5 applications of arbitrary 2-qubit gates $[81,82]$. These works have provided circuit representations using 7 applications of controlled-X rotation gates. We now use general techniques of Ref. [80] to recompile the controlled-sWAP gate and find more compact representations under the assumption that only a limited set of hardware-native gates can be executed by the hardware. We also find analytical guarantees that the recompiler has found the most compact representation possible. Results as the number $\nu_{e}$ of entangling gates and number $\nu_{s}$ of singlequbit gates are summarized in Table I. While the corresponding detailed circuits can be found online [58], we show the resulting circuit diagrams in Figs. 7-9.
TABLE I. Number $\nu_{e}$ of entangling gates with 2-(3-)qubit gates in bold (roman) and number $\nu_{s}$ of single-qubit gates when recompiling elementary controlled-swAP operations: fully equivalent (type A), local SU(4) equivalent recompilation (type B), and recompilation with including the observable $\sigma$ (type C). $R_{\alpha}$ denote single-qubit rotation gates with $\alpha \in\{x, y, z\}$, while $\mathrm{C}\left[R_{\alpha}\right]$ denote controlled rotations. $R_{x x}$ denotes the $x x$ gate and pSWAP is a parametrized SWAP gate. 3 -qubit gates in the last two rows are the $x x x$ gate $R_{x x x}$ and the controlled-controlled-phase gate $\mathrm{CC}[P]$. See Appendix E 1 for more details.

\begin{tabular}{lcrrrrr}
\hline \hline & \multicolumn{2}{c}{ Type $\mathrm{A}$} & \multicolumn{2}{c}{ Type B } & \multicolumn{2}{c}{ Type C } \\
Native gate set & $\nu_{e}$ & $\nu_{s}$ & $\nu_{e}$ & $\nu_{s}$ & $\nu_{e}$ & $\nu_{s}$ \\
\hline $\mathrm{C}\left[R_{x}\right], R_{y, z}$ & $\mathbf{6}$ & 6 & $\mathbf{5}$ & 2 & $\mathbf{4}$ & 2 \\
$\mathrm{C}\left[R_{z}\right], R_{x, y, z}$ & $\mathbf{6}$ & 15 & $\mathbf{5}$ & 4 & $\mathbf{4}$ & 4 \\
$R_{x x}, R_{y, z}$ & $\mathbf{6}$ & 11 & $\mathbf{5}$ & 6 & $\mathbf{4}$ & 6 \\
$\mathrm{pSWAP}, R_{x, y, z}$ & $\mathbf{6}$ & 11 & $\mathbf{5}$ & 4 & $\mathbf{4}$ & 3 \\
$R_{x x x}, R_{x x}, R_{y, z}$ & $3+\mathbf{3}$ & 10 & 3 & 6 & $2+\mathbf{1}$ & 6 \\
$\mathrm{CC}[P], R_{x, y}, \mathrm{C}\left[R_{z}\right]$ & $1+\mathbf{2}$ & 6 & $1+\mathbf{1}$ & 3 & $1+\mathbf{2}$ & 3 \\
\hline \hline
\end{tabular}

Equivalence classes.-Before discussing details of the recompilation, let us recapitulate basic definitions. A unitary $U$ is fully recompiled into $V$ if their action on every quantum state in the Hilbert space $\mathcal{H}$ is identical, i.e., there exists a global phase factor freedom $\exists \phi \in \mathbb{R}$ such that

$$
U|\psi\rangle=e^{-i \phi} V|\psi\rangle, \quad \forall \psi \in \mathcal{H} .
$$

We also consider the case of local SU(4) equivalent recompilations $V^{\prime}$ which, in contrast, result in equivalence only up to a local SU(4) transformation as

$$
\exists W \in \mathrm{SU}(4): U|\psi\rangle=e^{-i \phi} W V^{\prime}|\psi\rangle, \quad \forall \psi \in \mathcal{H} .
$$

We apply this definition to the case of elementary controlled-SWAP gates where the $\mathrm{SU}(4)$ transformation acts locally on the two swapped qubits. The reason why these circuits are important is the following. We notice that after the derangement circuit $D_{n}$ and the observable $\sigma$ in Fig. 1, we can apply any local unitary transformation $W$ to the quantum registers without changing the outcome of the measurement on the ancillary qubit. This generally allows us to recompile those controlled-SWAP gates into more compact circuits that are not followed by any further operations. As such, when considering $n=2$ copies, the entire derangement circuit can be recompiled into these more compact circuits. Let us now introduce the three types of recompilations used in this work.

Type A.-We consider the fully equivalent recompilation which may be necessary when $n>2$ and when implementing controlled-SWAP operations that are followed by other controlled-SWAP operators acting on the same registers. The first column of Table I shows that we generally need six 2-qubit operations to implement an elementary controlled- 
Type A: fully equivalent recompilation

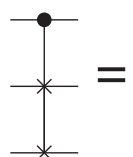

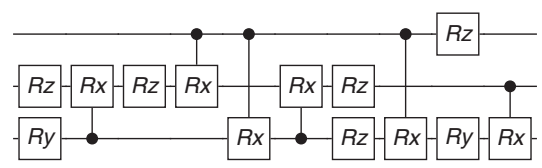

$\underset{*}{*}=$

$+\underset{+}{*}=$

$+\underset{*}{*}=$

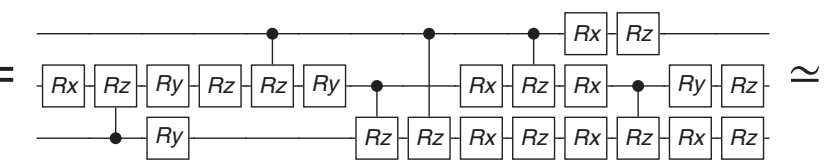

Type B: local SU(4)-equivalent recompilation

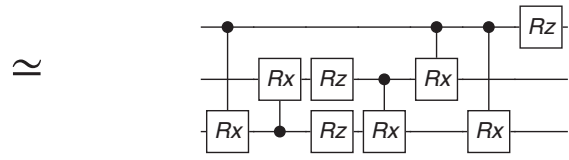

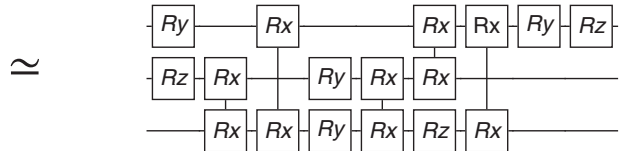
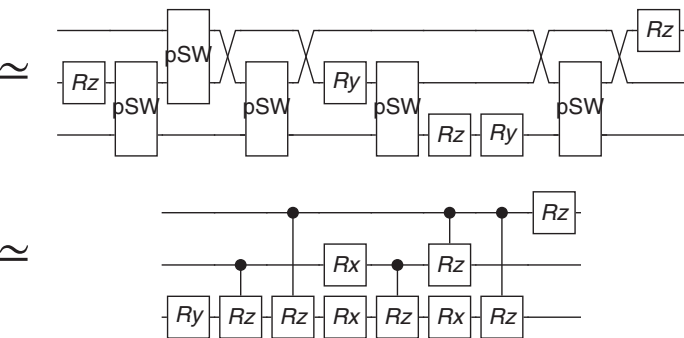

FIG. 7. Recompiling the controlled-SWAP gate into hardware-native gates. In all circuits the last $R_{z}$ rotation gate on the control qubit can be removed as it commutes with the controlled-swAP gate and can be merged with the basis transformation of the ancilla qubit (Hadamard gate) immediately prior to measurement in Fig. 1.

Type A: fully equivalent recompilation

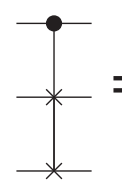

$+\frac{}{*}=$

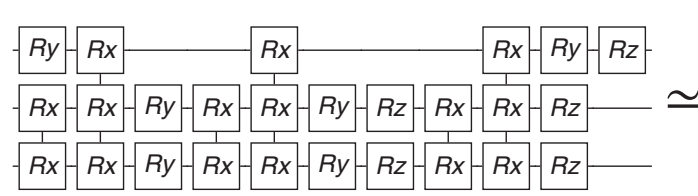

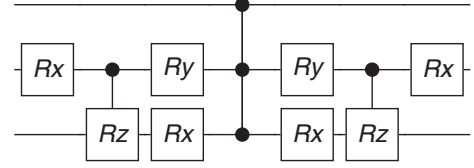

Type B: local SU(4)-equivalent recompilation
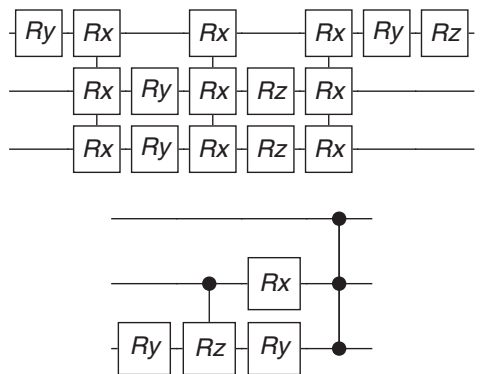

FIG. 8. Recompiling the controlled-SWAP gate assuming that the hardware can natively implement 3-qubit gates, such as the $x x x$ gate (first row) and the controlled-controlled-phase gate (second row).

Type C: recompilation with including the observable

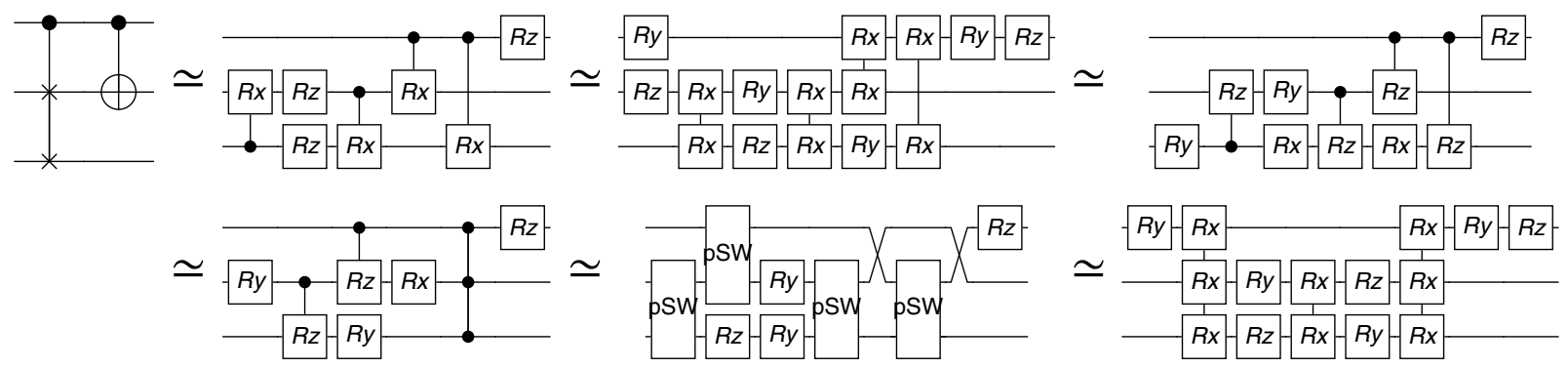

FIG. 9. Type C: recompiling the product of the controlled-swAP gate and the controlled observable $\sigma$ up to a local SU(4) freedom on the swapped qubits. 
SWAP gate, and corresponding compact circuits are illustrated in Figs. 7 and 8. We also need to consider fully equivalent recompilation if the observable is measured by postselecting on the ancilla and sampling the output of the registers-and not by implementing the controlled observable as in Fig. 1. The former scheme would allow us to estimate multiple observables simultaneously.

Type B.-If a controlled-swAP gate is not followed by any other gate, we only need to recompile the quantum gate up to a local SU(4) freedom. For example, in case of $n=3$ copies and observable $\sigma=\mathrm{Id}$, the derangement circuit consists of two swaps of pairs of registers as $\mathrm{C}\left[\mathrm{SWAP}_{2,3} \mathrm{SWAP}_{1,2}\right]$. We need to consider type A recompilation for $\mathrm{C}\left[\mathrm{SWAP}_{1,2}\right]$, and we can consider type $\mathrm{B}$ recompilation for $\mathrm{C}\left[\mathrm{SWAP}_{2,3}\right]$, since the latter is not followed by any other operation on the main registers. The second column of Table I shows that we generally need five 2-qubit operations to implement such an elementary gate. The resulting compact circuits are illustrated in Figs. 7 and 8 . We note that the entire $\mathrm{C}\left[\mathrm{SWAP}_{2,3} \mathrm{SWAP}_{1,2}\right]$ circuit could also be recompiled up to an $\mathrm{SU}(8)$ freedom.

Type C.-Observables as Pauli strings $\sigma \in$ $\left\{\mathrm{Id}_{2}, X, Y, Z\right\}^{\otimes N}$ act on some or all of the qubits nontrivially. For example, consider $n=2$ copies and the Pauli string $X_{4} Y_{9}$, in which case we can consider type $\mathrm{B}$ recompilation for all controlled-SWAP operators except for the ones that swap qubits 4 with $4^{\prime}$ and 9 with $9^{\prime}$ in the two registers. Similarly, we need only recompile the product of the elementary swap and the observable, $\mathrm{C}\left[X_{4} \mathrm{SWAP}_{4,4^{\prime}}\right]$ and $\mathrm{C}\left[Y_{9} \mathrm{SWAP}_{9,9^{\prime}}\right]$, up to a local $\mathrm{SU}(4)$ freedom. In the present work we fix an $X$ basis: one can thus implement $\mathrm{C}\left[\mathrm{SWAP}_{9,9^{\prime}} Y_{9}\right]$ by first transforming the basis of qubits 9 and $9^{\prime}$ using single-qubit rotations. The third column of Table I shows that we generally need four 2-qubit operations to implement such an elementary gate. The resulting compact circuits are illustrated in Fig. 9.

Gate sets.-Let us start by defining single-qubit $R_{\alpha}(\theta)$ rotation gates that depend on a parameter that can be calibrated to any fixed value in experiments $-2 \pi \leq \theta \leq 2 \pi$ as

$$
R_{\alpha}(\theta):=\exp \left[-i \frac{\theta}{2} \sigma_{\alpha}\right], \quad \text { with } \quad \sigma_{\alpha} \in\{X, Y, Z\},
$$

where $X, Y$, and $Z$ are Pauli matrices.

$\mathrm{C}\left[R_{X}\right]$ gates.-Let us first consider the aforementioned case of controlled- $X$ rotation gates. We assume that the hardware can natively implement single-qubit $Y$ and $Z$ rotations as well two-qubit controlled- $X$ rotations which we define as

$$
\mathrm{C}\left[R_{x}(\theta)\right]:=|0\rangle\left\langle 0\left|\otimes \operatorname{Id}_{2}+\right| 1\right\rangle\langle 1| \otimes R_{x}(\theta) .
$$

This unitary generates the CNOT gate when $\theta=\pi$. The controlled-SWAP gate can then be implemented via
6 (5) applications of the controlled- $x$ rotation gate in case of the fully (locally) equivalent recompilation as illustrated in the first row of Fig. 7. Refer to the last row of Fig. 7 for the similar case of controlled $R_{z}$ rotation gates.

$R_{x x}$ gates.-For the simulations in Fig. 4 we assume that the hardware can natively implement single-qubit $Y$ and $Z$ rotations as well as the 2-qubit $X X$ gate of the form

$$
R_{x x}(\theta):=\exp \left[-i \frac{\theta}{2} X \otimes X\right] \text {, }
$$

which generates the Mølmer-Sørensen gate at $\theta=-\pi / 2$. The controlled-SWAP gate can be implemented via 6 (5) applications of the XX rotation gate in case of the fully (locally) equivalent recompilation as illustrated in the second row of Fig. 7.

pSWAP gates.-We now consider an entangling gate that depends on two parameters $\theta_{1}$ and $\theta_{2}$ as

$\operatorname{pSWAP}\left[\theta_{1}, \theta_{2}\right]:=\exp \left[-i \frac{\theta_{1}}{2}(X \otimes X+Y \otimes Y)-i \frac{\theta_{2}}{2} Z \otimes Z\right]$,

which is typical to superconducting systems. This gate is locally equivalent to the fermionic simulation gate from Ref. [102] and generates at special angles many important gates, such as the SWAP gate. Although being a more general 2-qubit gate than the ones above, using the parametrized SWAP does not result in a significant improvement when recompiling the controlled-SWAP gate: We still need 6 (5) applications of the entangling gate in case of the fully (locally) equivalent recompilation as illustrated in the third row of Fig. 7.

$x x x$ gates. - Let us now turn to the question, can we obtain more compact representations of the controlledSWAP gate when we assume that the hardware can natively implement 3-qubit gates? Let us first consider the case when we allow both $x x$ and $x x x$ gates, where we define the latter as

$$
R_{x x x}(\theta):=\exp \left[-i \frac{\theta}{2} X \otimes X \otimes X\right]
$$

In this case we can analytically solve the recompilation problem by recalling that the controlled-SWAP gate can be expressed as

$$
\begin{aligned}
\mathrm{C}[\mathrm{SWAP}]= & \exp \left[-i \frac{\pi}{8}\left(\mathrm{Id}_{2}-Z\right) \otimes(X \otimes X+Y \otimes Y\right. \\
& \left.\left.+Z \otimes Z-\mathrm{Id}_{2} \otimes \mathrm{Id}_{2}\right)\right] .
\end{aligned}
$$

Since above all terms in the exponential commute, we can express this gate as the following series of gates executed in arbitrary order: a $z x x$ gate, a $z y y$ gate, a $z z z$ gate, an $x x$ gate, a $y y$ gate, a $z z$ gate, and additionally a $z$ gate on the ancilla, which however can be removed as discussed below Fig. 7 . 
Indeed, our recompiler has found exactly this kind of circuit by mapping the $x x x$ gate to, e.g., the $z y y$ gate via single-qubit rotations. Refer to the first row of Fig. 8. Since all these multiqubit gates commute, we can order them such that all the $x x$, the $y y$, and the $z z$ gates are at the end of the circuit. These gates then form a local SU(4) unitary that can be removed as discussed above. Indeed, our recompiler has found this solution when considering only locally equivalent recompilations as illustrated in Fig. 8.

$\mathrm{CC}[P]$ gates.-Let us finally consider controlled-controlled phase gates, which have been successfully implemented in experiments as native gates [103]. Let us define this gate as

$$
\mathrm{CC}[P]:=\operatorname{diag}(1,1,1,1,1,1,1,-1) .
$$

These allow for surprisingly compact representations: the controlled-SWAP gate can be implemented using a single application of the $\mathrm{CC}[P]$ gate plus 2 (1) applications of controlled-Z rotation gates as illustrated in the second row of Fig. 8.

\section{Exploiting symmetries in derangement circuits}

As discussed in the main text, derangement circuits have a rapidly growing number of invariants when increasing the number of copies $n$ or the number of qubits $N$. This includes the large number of distinct permutations from Definition 1. Let us illustrate how these symmetries can be exploited via the following three examples.

Example 1: In the first example we assume that the connectivity between registers is limited such that only nearest-neighbor registers can be swapped. For example, this could be a quantum device with $n$ individual quantum processors arranged in a line. A derangement operation in this case is preferred that swaps only nearest-neighbor registers. For example, for $n=4$ we can first apply nearestneighbor SWAP operators between registers as $\mathrm{SWAP}_{1,2}$ and $\mathrm{SWAP}_{3,4}$ followed by SWAP 2,3 . In contrast, a derangement which uses, e.g., $\mathrm{SWAP}_{1,4}$ would not be supported natively by the device. Refer to Ref. [58] for a demonstration of the various distinct derangement circuits.

Example 2: In the second example we assume that arbitrary connectivity is available. In this case one can in principle implement any of the distinct derangement (permutation) patterns. Although choosing and fixing any one of those is sufficient, it is also possible to randomly choose from these circuits since noise may affect them differently. This may also help in suppressing asymmetries in the potentially nonidentical input density matrices thereby creating randomized, "average" density matrices when $n$ is large.

Note that further invariants exist due to the fact that the $\sigma$ gate in Fig. 1 can be applied to any of the registers.

Example 3: In the third example let us consider an approach that can exploit symmetries in the derangement operator in complete analogy with twirling techniques. We notice that the controlled-derangement operator is a very specific kind of operation: when the ancilla state is $|0\rangle$ then the registers are left invariant and when the ancilla state is $|1\rangle$ then the registers are permuted. We start by applying an operation $U$ before the derangement operator; i.e., Pauli strings $P_{k} \in\left\{\mathrm{Id}_{2}, X, Y, Z\right\}^{\otimes N}$ are applied to registers $k$ with $U:=\bigotimes_{k=1}^{n} P_{k}$. We can undo this operation after the derangement operator by first applying the anticontrolled Pauli string $U$. This is then followed by the controlled Pauli string $U^{\prime}$, where in $U^{\prime}$ we need to relabel the indices $k$ according to the permutation $s(k)$ that the derangement operator implements, i.e., $U^{\prime}:=\bigotimes_{k=1}^{n} P_{s(k)}$.

Applying the above (controlled) Pauli strings before (after) the controlled-derangement operator does not affect the expectation-value measurement in an ideal scenario. However, the Pauli strings in $U$ applied to the registers do reflect the errors that happen during the swap process; thus randomly applying Pauli strings and averaging the measurement results can reduce and homogenize the impact of errors that happen in the derangement circuit. Note that this is analogous to twirling techniques.

Let us illustrate this on the particular case of $n=2$ copies. We randomly select two Pauli strings, $P_{1}, P_{2} \in$ $\left\{\mathrm{Id}_{2}, X, Y, Z\right\}^{\otimes N}$, where we apply $P_{1}$ to the first input state $\rho$ and apply $P_{2}$ to the second register. Note that these are just single-qubit $X, Y$, and $Z$ operations (or the identity) applied to individual qubits in the registers. We then perform the controlled-derangement operator $D_{2}$ which swaps the two registers. We can now undo the effect of Pauli strings by applying the anticontrolled Pauli string $P_{1}$ and $P_{2}$ to registers 1 and 2, respectively. Since the derangement operator swaps the two registers, we apply the controlled $P_{1}$ operator to the second register and the controlled $\mathrm{P}_{2}$ operator to the first register.

We have simulated the above example assuming a system of $N=3,4$, and 5 qubits using recompiled controlled-SWAP operators from Appendix F 3. We assume the noise model and the same native gate set as in Appendix F 3. We randomly generate input states $\rho$ and compute the error in the derangement circuit as $\mathcal{E}=\operatorname{Tr}\left[\rho^{2} \sigma\right] / \operatorname{Tr}\left[\rho^{2}\right]-\left(2 \operatorname{prob}_{0}-1\right) /$ $\left(2 \operatorname{prob}_{0}^{\prime}-1\right)$, where we estimate the probabilities prob $_{0}$ and $\operatorname{prob}_{0}^{\prime}$ using the circuit in Fig. 1. We then randomly select and apply 50 pairs of Pauli strings $P_{1}$ and $P_{2}$ and average the estimated probabilities; we denote the resulting errors as $\tilde{\mathcal{E}}$. The ratio of the errors with and without twirling $F:=\tilde{\mathcal{E}} / \mathcal{E}$ is plotted in Fig. 10 (left). Note that the controlled Pauli strings $P_{1}$ and $P_{2}$ introduce additional noise when compared to just applying the plain controlled-derangement operator. Nevertheless, Fig. 10 (left) highlights that the above twirling scheme is still able to reduce $30 \%-50 \%$ of errors in the practically most important region, i.e., when the circuit error rate is $\xi<2$.

It is important to note that the simulations in Fig. 10 (left) should be viewed as a worst-case scenario for the following reasons. (a) In the example we have considered $n=2$ 

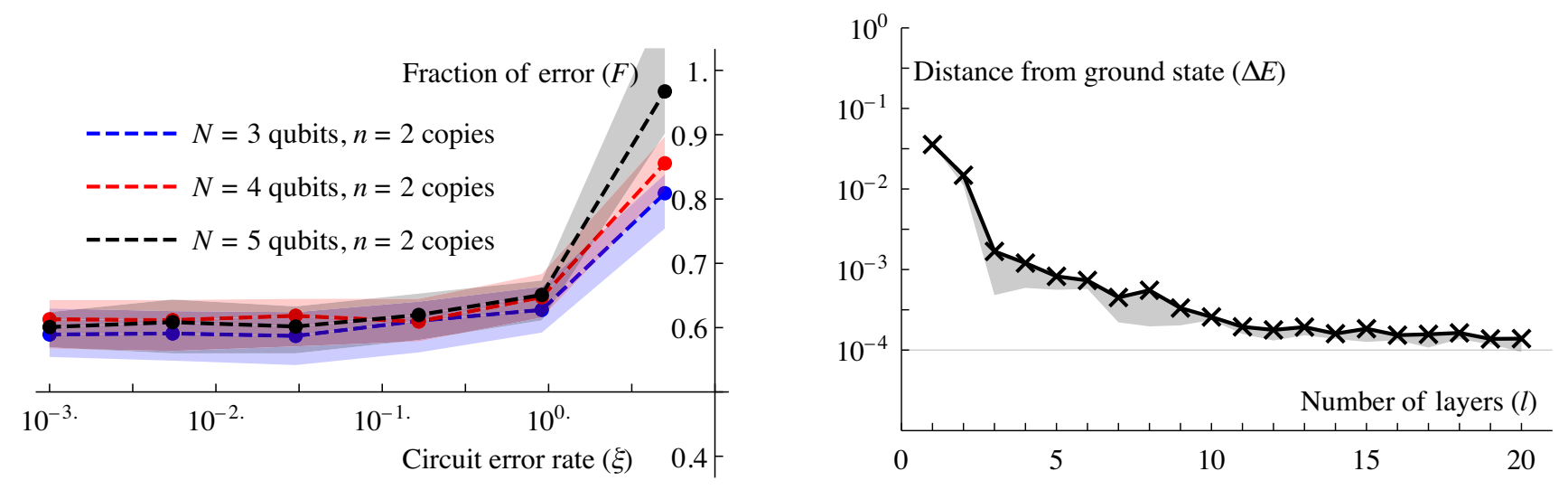

FIG. 10. Left: reducing errors in the derangement operator $D_{2}$ via generalized twirling operations as discussed in Appendix E 2. Errors in measuring expectation values of 50 randomly selected observables $\sigma \in\left\{\operatorname{Id}_{2}, X, Y, Z\right\}^{\otimes N}$ were computed with $(\tilde{\mathcal{E}})$ and without $(\mathcal{E})$ twirling. The median of the ratios $F$ of the two errors $F:=\tilde{\mathcal{E}} / \mathcal{E}$ is plotted as a function of the number of expected errors in the derangement circuit $(\xi)$ - shading represents the quantiles $1 / 4$ and $3 / 4$. Twirling reduces $30 \%-50 \%$ of errors in the noisy derangement circuit, but it can be viewed as a worst-case scenario as discussed in Appendix E 2. Right: finding the ground-state energy $E$ of the spinring Hamiltonian from Eq. (8) using the variational Hamiltonian Ansatz with $l$ layers as discussed in Appendix F 3. The Ansatz parameters were optimized in 5 independent instances and the average of the distance from the ground-state energy $\Delta E$ is plotted as a function of $l$. Shading represents the minimum of the 5 instances.

copies, in which case we need 6 entangling gates per qubit in a register to fully recompile controlled-SWAP operators. In the simulations we naively implemented the twirling technique resulting in 2 additional entangling gates per qubit. When we consider a larger number of copies, e.g., $n=5$, the overhead of the twirling technique remains 2 entangling gates, but implementing the derangement operation requires proportionally more entangling gates. This leads to a decreasing overhead of the twirling technique. (b) As discussed above, the controlled observable can be combined with elementary controlled-SWAP gates via recompilation resulting in no increase in the number of 2-qubit gates in the derangement circuit. Similarly, we can recompile the entire, twirled controlled-SWAP operator into one compact circuit. This will significantly reduce the gatecount overhead of the twirled circuit thereby increasing the efficacy of the error reduction factor $F$.

\section{APPENDIX F: NUMERICAL SIMULATIONS}

\section{Simulations in Fig. 2}

We consider an alternating-layer Ansatz with 10 layers and 12 qubits as illustrated in Fig. 11. The circuit consists of overall 372 noisy gates and each 2-qubit gate undergoes 2-qubit depolarizing noise with $0.5 \%$ probability while each single-qubit gate undergoes depolarizing noise with $0.05 \%$ probability. Each gate is parametrized and we have selected their parameters randomly.

We computed the density matrix of a single copy of the state $\rho$ and our derangement circuit uses $n$ copies of this state as input. While we have numerically verified full derangement circuits with smaller density matrices, here we aim to efficiently compute approximation errors. In particular, we only need to store a single copy of $\rho$ and perform computations to obtain $\operatorname{Tr}\left[\rho^{n} \sigma\right]$ and $\operatorname{Tr}\left[\rho^{n}\right]$ for randomly

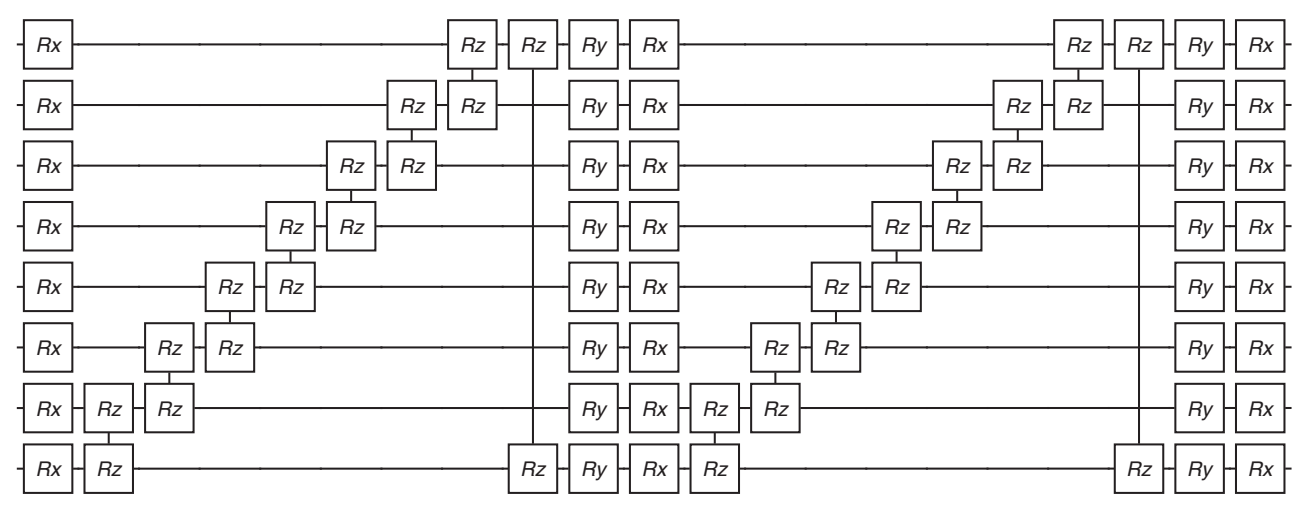

FIG. 11. Example of a 2-block Ansatz circuit of 8 qubits. We used a 10-block circuit of 12 qubits in our simulations in Fig. 2 consisting of overall 372 noisy gates. Each 2-qubit gate undergoes 2-qubit depolarizing noise with $0.5 \%$ probability and each single-qubit gate undergoes depolarizing noise with $0.05 \%$ probability. 
selected Pauli strings $\sigma$. Furthermore, we diagonalize $\rho$ and use its eigenvalues for computing Rényi entropies exactly while we use its dominant eigenvector $|\psi\rangle$ to determine the expectation value $\langle\psi|\sigma| \psi\rangle$.

We remark here that we compute approximation errors as the deviation from the expectation value obtained from the dominant eigenvector $\langle\psi|\sigma| \psi\rangle$ and do not directly compare to noise-free computations due to the coherent mismatch discussed in Appendix C 1 (which becomes negligible for large systems and can be addressed with standard techniques). In our simulations this coherent mismatch was below $10^{-4}$, and could be corrected with usual techniques that aim to suppress coherent errors as discussed in the main text.

\section{Simulations in Fig. 3}

We have simulated a derangement circuit that takes $n=3$ copies of a noisy 4-qubit state as input and the controlledSWAP operators also undergo depolarizing noise (with a probability of $10^{-3}$ ) as shown in Fig. 12. The input state is produced by a parametrized 4-qubit circuit and we have selected 50 sets of parameters randomly and performed extrapolation techniques on each instance as shown in Fig. 3.

\section{Ground-state simulation in Fig. 4}

We consider the spin-ring Hamiltonian in Eq. (8) and aim to determine its ground state using the variational Hamiltonian Ansatz [11-13,22,64,74-78]. The Ansatz consists of alternating layers of time evolutions under the Hamiltonians which we define as

$$
\mathcal{H}_{0}:=\sum_{k=1}^{N} \omega_{k} Z_{k} \quad \text { and } \quad \mathcal{H}_{1}:=J \sum_{k \in \operatorname{ring}(N)} \vec{\sigma}_{k} \cdot \vec{\sigma}_{k+1},
$$

via $\mathcal{H}=\mathcal{H}_{0}+\mathcal{H}_{1}$, as illustrated in Fig. 4(a). We can analytically determine and start the optimization from the ground state $\left|\psi_{\text {init }}\right\rangle$ of $\mathcal{H}_{0}$ as a computational basis state as in, e.g., Ref. [74]. We then apply alternating layers of the parametrized evolutions $A\left(\gamma_{k}\right):=e^{-i \gamma_{k} \mathcal{H}_{1}}$ and $B\left(\beta_{k}\right):=e^{-i \beta_{k} \mathcal{H}_{0}}$ to this initial state as

$$
|\psi(\underline{\beta}, \underline{\gamma})\rangle=B\left(\beta_{l}\right) A\left(\gamma_{l}\right) \ldots A\left(\gamma_{2}\right) B\left(\beta_{1}\right) A\left(\gamma_{1}\right)\left|\psi_{\text {init }}\right\rangle,
$$

using overall $l$ layers. The parameters $\beta$ and $\gamma$ are optimized by a classical coprocessor such that the estimated energy
$E:=\operatorname{Tr}[\rho \mathcal{H}]$ is minimized. We consider a quantum device that can natively implement single-qubit $R_{y}$ and $R_{z}$ rotation gates as well as XX gates of the form $\exp \left[-i \theta X_{j} X_{k}\right]$ between any pairs $j \neq k$ of qubits. This gate set is comparable to iontrap systems [79] and the above discussed Ansatz can be implemented efficiently the following way. The evolution under $\mathcal{H}_{1}$ is Trotterized such that every term in the Hamiltonian is implemented independently via a gate of the form, e.g., $\exp \left[-i \theta X_{j} X_{k}\right]$. Single-qubit rotations are used to implement gates of the form $\exp \left[-i \theta Z_{j} Z_{k}\right]$ by rotating the $Z$ basis to an $X$ basis. It follows that the Ansatz circuit with $l$ layers can be implemented via $3 \mathrm{Nl}$ applications of the $X X$ entangling gates.

The number of layers $l$ to reach a given precision with respect to the ground state depends on the particular Hamiltonian and on the number of qubits [63,78]. We fix every degree of freedom and determine the number of layers required to reach a difference $\Delta E=10^{-4}$ to the ground-state energy. We set $N=6$ qubits, choose a coupling constant $J=0.1$, and randomly generate and fix the on-site energies as $\quad \underline{\omega}=(-0.70983,-0.0517,0.9065,-0.9265,0.0950$, -0.49597). We optimize the Ansatz parameters $\underline{\beta}$ and $\underline{\gamma}$ by applying 1000 iterations of natural gradient evolution to a set of randomly chosen initial parameters in the vicinity of the parameters that approximate the adiabatic evolution. We perform 5 independent optimizations and plot the average and the minimum of the distance $\Delta E$ in Fig. 10 (right). The average and the minimum follow the expected scaling $[63,78]$ and the minimum reaches $\Delta E=10^{-4}$ at $l=20$ layers. Furthermore, regarding the Ansatz depth we find that $l=20$ is comparable to results of Refs. [62,63,78]. It follows that we can implement the Ansatz circuit with $3 N l=360$ applications of the native entangling gate. Let us remark that even though one may be able to find more compact Ansätze [104], the variational Hamiltonian Ansatz has the strong benefit of being informed by the problem structure - and it is guaranteed to find the ground state for an increasing depth due to its convergence to an adiabatic evolution [22]. Furthermore, even when using more compact Ansätze, it is generally expected that the depth of the computation needs to grow when increasing the scale of the computation as discussed in the main text.

We assume the following noise model. Single-qubit gates are followed by dephasing noise with probability $\epsilon$ and damping (relaxation) noise with a small probability $0.1 \epsilon$. We also assume that the experimentalist can amplify

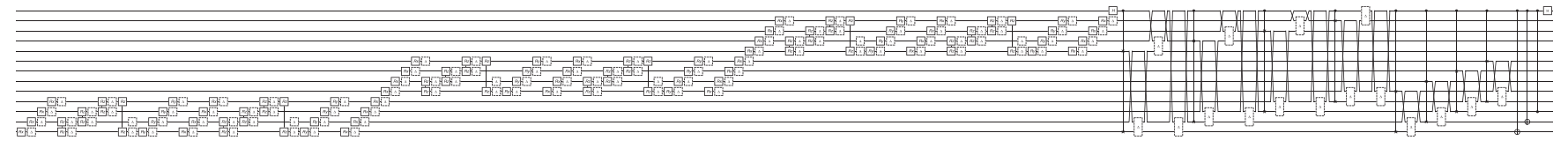

FIG. 12. Circuit that we simulate in Fig. 3. A controlled-swAP gate acting on qubits $k, l$, and $m$ is followed by 2-qubit depolarizations between qubits $k, l$ and $k, m$ and $l, m$. A variant in which damping errors follow the depolarizations effectively resulted in the same error mitigation performance. 
this noise by increasing the value of $\epsilon$. Furthermore, the qubits also undergo a small depolarizing noise with probability $0.07 \epsilon$, but we assume this noise cannot be amplified by the experimentalist. The ratio of the nonextrapolatable noise to the total gate error rate can be expressed via the probabilities

$$
\begin{aligned}
& \frac{\operatorname{prob}(\text { nonextrapolatable error })}{\operatorname{prob}(\text { error })} \\
& =\frac{0.07 \epsilon}{1-(1-\epsilon)(1-0.1 \epsilon)(1-0.07 \epsilon)} \\
& \approx 0.06+0.009 \epsilon+\mathcal{O}\left(\epsilon^{2}\right) .
\end{aligned}
$$

We assume the same noise model in the case of 2-qubit gates but with all probabilities magnified by a factor of 5 , i.e., $\epsilon \rightarrow 5 \epsilon$.

In selecting an error model, it was important to include key characteristics of real systems while retaining the ability to perform efficient simulations. The model chosen exhibits the key elements of dephasing, damping, and depolarizing, and therefore captures the core characteristics of systems such as ion traps, encompassing finite $T 2$ relaxation (via dephasing), $T 1$ relaxation (via damping), and imperfect control, heating, etc., (via depolarization). It is typical in ion traps, superconducting systems, and other platforms that single-qubit gate infidelities are significantly less severe than those of 2-qubit gates and therefore this characteristic was incorporated. Moreover, no real system can be expected to support perfect extrapolation, as this implies flawless scaling of all error contributions; here the chosen model makes the assumption that the nonextrapolatable component is small; this is favorable to established extrapolation techniques and therefore provides a rigorous test for our new protocol.

While the resulting comparison is therefore physically plausible, it is worth noting that the ESD technique should also be expected to be robust over a wide variety of other noise models. Specifically, the theoretical error bounds in result 1 depend only on the eigenvalue distribution of the density matrix; indeed, additional simulations were performed (not reported here) using a depolarizing noise model that confirm these theoretical expectations.

In the present example we consider $N=6$ qubits and $n=2$ copies; i.e., we need to simulate the density matrix of 13 qubits which is equivalent to a 26-qubit pure-state simulation. We recompile the derangement circuit into hardware-native gates as discussed in Appendix E 1: we only need to recompile the elementary controlled-SWAP gates up to a local SU(4) freedom (that acts on the swapped qubits). When estimating the probability prob $_{0}^{\prime}$ we thus need to use type B circuits from the second column of Table I; see also second column in Fig 7. Implementing the corresponding derangement circuit thus requires overall $5 \mathrm{~N}$ applications of the entangling gate. When estimating the probability prob $_{0}$ we either use circuits of type $\mathrm{B}$ or type $\mathrm{C}$ depending on whether the observable acts on the particular qubit. For example, when estimating the expectation value of the observable $Z_{1}$, we use type $\mathrm{B}$ circuits for all controlled-sWAP gates except the one that swaps the first qubits in both registers as $\mathrm{SWAP}_{1,1^{\prime}}$. For the latter we use the type $\mathrm{C}$ circuit after rotating the basis of qubits 1 and $1^{\prime}$ so that the observable is effectively mapped $Z_{1} \rightarrow X_{1}$; refer to the third column in Table I and to Fig 9. Thus estimating the probability prob $_{0}$ for a single-qubit observable requires $5(N-1)+4=29$ applications of the native entangling gates while in the case of 2-qubit observables we need $5(N-2)+8=28$ entangling gates. Note that estimating expectation values of nonlocal observables as $\sigma \in\{X, Y, Z\}^{\otimes N}$ would be more noise robust as we would only need $4 N$ entangling gates to implement the derangement circuit.

Orange diamonds in Fig. 4(c) show the performance of zero-noise extrapolation using a polynomial fitting: this technique reduced close to $85 \%$ of errors in the region where the circuit error rate is not too large, i.e., $\xi<1$. Linear and exponential fits were also implemented, but polynomial fitting slightly outperformed exponential and linear fits. Magenta dots in Fig. 4(c) show the performance of the noisy derangement circuit. Errors in the derangement circuit were amplified and extrapolated using polynomial fitting; see black crosses in Fig. 4(c). The extrapolation significantly reduces errors in the derangement circuit, thus closely approximating the performance of noiseless derangement circuits (dashed blue line). Note that even without extrapolation, the derangement circuit can reduce the errors by orders of magnitude; i.e., compare red squares with magenta dots. In all cases polynomial fitting was preformed via a least squares fitting of a degree 3 polynomial using 6 estimated points in the interval between $\epsilon$ and $2 \epsilon$.

Let us finally illustrate how connectivity constraints may affect the ESD approach via the following simple example. Let us assume that qubits form a $2 \times(N+1)$ array and nearest-neighbor interactions are possible. The qubits in positions $(0,1 \rightarrow N)$ and $(1,1 \rightarrow N)$ are assigned to two copies of the $N$-qubit state, while we assign the qubit $(0,0)$ to the ancilla. Once the main computation is done, one can implement the controlled-SWAP operator between qubits $(0,1)$ and $(1,1)$ controlled on the ancilla $(0,0)$ using only nearest-neighbor interactions via Table I. We then move the ancilla to the next position by swapping qubits $(0,0)$ and $(0,1)$. This now allows us to apply the next controlled-SWAP operator between qubits $(0,2)$ and $(1,2)$ controlled on the ancilla $(0,1)$. Repeating this procedure for all qubits in the registers allows us to implement the derangement circuit with an overhead of only 1 extra 2-qubit SWAP gate per controlled-SWAP operation. It is straightforward to generalize this idea to arbitrary numbers of copies or to "parallelizing" the process by distributing the ancilla 
among many qubits via a Greenberger-Horne-Zeilinger (GHZ) state. On the other hand, when implementing the Ansatz circuit from Fig. 4, one needs to apply $2 N$ swAP gates to be able to entangle the first and last qubits in the register as required for the spin-ring Hamiltonian. This increases the number of entangling gates in the Ansatz circuit from $3 \mathrm{Nl}$ to $9 \mathrm{Nl}$. Thus, in such a scenario connectivity constraints would work in our favor.

[1] M. A. Nielsen and I. L. Chuang, Quantum Computation and Quantum Information, 10th ed. (Cambridge University Press, New York, 2011).

[2] D. A. Lidar and T. A. Brun, Quantum Error Correction (Cambridge University Press, Cambridge, England, 2013).

[3] D. Gottesman, Theory of Fault-Tolerant Quantum Computation, Phys. Rev. A 57, 127 (1998).

[4] P. W. Shor, Scheme for Reducing Decoherence in Quantum Computer Memory, Phys. Rev. A 52, R2493 (1995).

[5] A. R. Calderbank and P. W. Shor, Good Quantum ErrorCorrecting Codes Exist, Phys. Rev. A 54, 1098 (1996).

[6] E. Knill, R. Laflamme, and W. H. Zurek, Resilient Quantum Computation, Science 279, 342 (1998).

[7] D. Aharonov and M. Ben-Or, Fault-Tolerant Quantum Computation with Constant Error, in Proceedings of the Twenty-Ninth Annual ACM Symposium on Theory of Computing, STOC '97 (Association for Computing Machinery, New York, 1997), p. 176188.

[8] J. Preskill, Quantum Computing in the NISQ Era and Beyond, Quantum 2, 79 (2018).

[9] R. Laflamme, C. Miquel, J. P. Paz, and W. H. Zurek, Perfect Quantum Error Correcting Code, Phys. Rev. Lett. 77, 198 (1996).

[10] C. H. Bennett, D. P. DiVincenzo, J. A. Smolin, and W. K. Wootters, Mixed-State Entanglement and Quantum Error Correction, Phys. Rev. A 54, 3824 (1996).

[11] M. Cerezo, A. Arrasmith, R. Babbush, S. C. Benjamin, S. Endo, K. Fujii, J. R. McClean, K. Mitarai, X. Yuan, L. Cincio et al., Variational Quantum Algorithms, arXiv: 2012.09265.

[12] S. Endo, Z. Cai, S. C. Benjamin, and X. Yuan, Hybrid Quantum-Classical Algorithms and Quantum Error Mitigation, J. Phys. Soc. Jpn. 90, 032001 (2021).

[13] K. Bharti, A. Cervera-Lierta, T. H. Kyaw, T. Haug, S. Alperin-Lea, A. Anand, M. Degroote, H. Heimonen, J. S. Kottmann, T. Menke et al., Noisy Intermediate-Scale Quantum (NISQ) Algorithms, arXiv:2101.08448.

[14] Y. Li and S. C. Benjamin, Efficient Variational Quantum Simulator Incorporating Active Error Minimization, Phys. Rev. X 7, 021050 (2017).

[15] S. Endo, S. C. Benjamin, and Y. Li, Practical Quantum Error Mitigation for Near-Future Applications, Phys. Rev. X 8, 031027 (2018).

[16] A. Kandala, K. Temme, A. D. Córcoles, A. Mezzacapo, J. M. Chow, and J. M. Gambetta, Error Mitigation Extends the Computational Reach of a Noisy Quantum Processor, Nature (London) 567, 491 (2019).
[17] K. Temme, S. Bravyi, and J. M. Gambetta, Error Mitigation for Short-Depth Quantum Circuits, Phys. Rev. Lett. 119, 180509 (2017).

[18] A. Strikis, D. Qin, Y. Chen, S. C. Benjamin, and Y. Li, Learning-Based Quantum Error Mitigation, arXiv: 2005.07601.

[19] P. Czarnik, A. Arrasmith, P. J. Coles, and L. Cincio, Error Mitigation with Clifford Quantum-Circuit Data, arXiv: 2005.10189.

[20] S. McArdle, X. Yuan, and S. Benjamin, Error-Mitigated Digital Quantum Simulation, Phys. Rev. Lett. 122, 180501 (2019).

[21] A. G. Rattew, Y. Sun, P. Minssen, and M. Pistoia, Quantum Simulation of Galton Machines Using Mid-Circuit Measurement and Reuse, arXiv:2009.06601.

[22] E. Farhi, J. Goldstone, and S. Gutmann, A Quantum Approximate Optimization Algorithm, arXiv:1411.4028.

[23] A. Peruzzo, J. McClean, P. Shadbolt, M.-H. Yung, Q. Zhou, P. J. Love, A. Aspuru-Guzik, and J. L. O'Brien, A Variational Eigenvalue Solver on a Photonic Quantum Processor, Nat. Commun. 5 (2014).

[24] Y. Wang, F. Dolde, J. Biamonte, R. Babbush, V. Bergholm, S. Yang, I. Jakobi, P. Neumann, A. Aspuru-Guzik, J. D. Whitfield et al., Quantum Simulation of Helium Hydride Cation in a Solid-State Spin Register, ACS Nano 9, 7769 (2015).

[25] P. J. J. O’Malley, R. Babbush, I. D. Kivlichan, J. Romero, J. R. McClean, R. Barends, J. Kelly, P. Roushan, A. Tranter, N. Ding et al., Scalable Quantum Simulation of Molecular Energies, Phys. Rev. X 6, 031007 (2016).

[26] Y. Shen, X. Zhang, S. Zhang, J.-N. Zhang, M.-H. Yung, and K. Kim, Quantum Implementation of the Unitary Coupled Cluster for Simulating Molecular Electronic Structure, Phys. Rev. A 95, 020501(R) (2017).

[27] J. R. McClean, J. Romero, R. Babbush, and A. AspuruGuzik, The Theory of Variational Hybrid QuantumClassical Algorithms, New J. Phys. 18, 023023 (2016).

[28] S. Paesani, A. A. Gentile, R. Santagati, J. Wang, N. Wiebe, D. P. Tew, J. L. O'Brien, and M. G. Thompson, Experimental Bayesian Quantum Phase Estimation on a Silicon Photonic Chip, Phys. Rev. Lett. 118, 100503 (2017).

[29] J. I. Colless, V. V. Ramasesh, D. Dahlen, M. S. Blok, M. E. Kimchi-Schwartz, J. R. McClean, J. Carter, W. A. de Jong, and I. Siddiqi, Computation of Molecular Spectra on a Quantum Processor with an Error-Resilient Algorithm, Phys. Rev. X 8, 011021 (2018).

[30] R. Santagati, J. Wang, A. A. Gentile, S. Paesani, N. Wiebe, J. R. McClean, S. Morley-Short, P. J. Shadbolt, D. Bonneau, J. W. Silverstone, D. P. Tew, X. Zhou, J. L. O'Brien, and M. G. Thompson, Witnessing Eigenstates for Quantum Simulation of Hamiltonian Spectra, Sci. Adv. 4, eaap9646 (2018).

[31] A. Kandala, A. Mezzacapo, K. Temme, M. Takita, M. Brink, J. M. Chow, and J. M. Gambetta, HardwareEfficient Variational Quantum Eigensolver for Small Molecules and Quantum Magnets, Nature (London) 549, 242 (2017).

[32] C. Hempel, C. Maier, J. Romero, J. McClean, T. Monz, H. Shen, P. Jurcevic, B. P. Lanyon, P. Love, R. Babbush, A. 
Aspuru-Guzik, R. Blatt, and C. F. Roos, Quantum Chemistry Calculations on a Trapped-Ion Quantum Simulator, Phys. Rev. X 8, 031022 (2018).

[33] J. Romero, R. Babbush, J. R. McClean, C. Hempel, P. J. Love, and A. Aspuru-Guzik, Strategies for Quantum Computing Molecular Energies Using the Unitary Coupled Cluster Ansatz, Quantum Sci. Technol. 4, 014008 (2018).

[34] O. Higgott, D. Wang, and S. Brierley, Variational Quantum Computation of Excited States, Quantum 3, 156 (2019).

[35] J. R. McClean, M. E. Kimchi-Schwartz, J. Carter, and W. A. de Jong, Hybrid Quantum-Classical Hierarchy for Mitigation of Decoherence and Determination of Excited States, Phys. Rev. A 95, 042308 (2017).

[36] J. I. Colless, V. V. Ramasesh, D. Dahlen, M. S. Blok, J. R. McClean, J. Carter, W. A. de Jong, and I. Siddiqi, Robust Determination of Molecular Spectra on a Quantum Processor, Phys. Rev. X 8, 011021 (2018).

[37] C. Kokail, C. Maier, R. van Bijnen, T. Brydges, M. K. Joshi, P. Jurcevic, C. A. Muschik, P. Silvi, R. Blatt, C.F. Roos et al., Self-Verifying Variational Quantum Simulation of Lattice Models, Nature (London) 569, 355 (2019).

[38] K. Sharma, S. Khatri, M. Cerezo, and P. J. Coles, Noise Resilience of Variational Quantum Compiling, New J. Phys. 22, 043006 (2020).

[39] B. Koczor, S. Endo, T. Jones, Y. Matsuzaki, and S. C. Benjamin, Variational-State Quantum Metrology, New J. Phys. 22, 083038 (2020).

[40] B. Koczor and S. C. Benjamin, Quantum Natural Gradient Generalised to Non-Unitary Circuits, arXiv:1912.08660.

[41] B. Koczor and S. C. Benjamin, Quantum Analytic Descent, arXiv:2008.13774.

[42] X. Yuan, S. Endo, Q. Zhao, Y. Li, and S. C. Benjamin, Theory of Variational Quantum Simulation, Quantum 3, 191 (2019).

[43] O. Crawford, B. van Straaten, D. Wang, T. Parks, E. Campbell, and S. Brierley, Efficient Quantum Measurement of Pauli Operators, Quantum 5, 385 (2021).

[44] C. Hadfield, S. Bravyi, R. Raymond, and A. Mezzacapo, Measurements of Quantum Hamiltonians with LocallyBiased Classical Shadows, arXiv:2006.15788.

[45] B. Koczor, The Dominant Eigenvector of a Noisy Quantum State, arXiv:2104.00608.

[46] A. Rényi, On Measures of Information and Entropy, in Proceedings of the 4th Berkeley Symposium on Mathematics, Statistics and Probability (University of California Press, Berkeley and Los Angeles, 1961), Vol. 1, pp. 547561.

[47] A. K. Ekert, C. M. Alves, D. K. L. Oi, M. Horodecki, P. Horodecki, and L. C. Kwek, Direct Estimations of Linear and Nonlinear Functionals of a Quantum State, Phys. Rev. Lett. 88, 217901 (2002).

[48] M. Keyl and R. F. Werner, Estimating the Spectrum of a Density Operator, Phys. Rev. A 64, 052311 (2001).

[49] I. Marvian and R. W. Spekkens, A Generalization of Schur-Weyl Duality with Applications in Quantum Estimation, Commun. Math. Phys. 331, 431 (2014).
[50] J. Acharya, I. Issa, N. V. Shende, and A. B. Wagner, Estimating Quantum Entropy, IEEE J. Sel. Areas Inf. Theory 1, 454 (2020).

[51] T. Tanaka, Y. Ota, M. Kanazawa, G. Kimura, H. Nakazato, and F. Nori, Determining Eigenvalues of a Density Matrix with Minimal Information in a Single Experimental Setting, Phys. Rev. A 89, 012117 (2014).

[52] M. Christandl, A. W. Harrow, and G. Mitchison, Nonzero Kronecker Coefficients and What They Tell Us about Spectra, Commun. Math. Phys. 270, 575 (2007).

[53] M. Christandl and G. Mitchison, The Spectra of Quantum States and the Kronecker Coefficients of the Symmetric Group, Commun. Math. Phys. 261, 789 (2006).

[54] R. Harper, W. Yu, and S. T. Flammia, Fast Estimation of Sparse Quantum Noise, PRX Quantum 2, 010322 (2021).

[55] B. van Straaten and B. Koczor, Measurement Cost of Metric-Aware Variational Quantum Algorithms, PRX Quantum 2, 030324 (2021).

[56] F. Roberts and B. Tesman, Applied Combinatorics (CRC Press, Boca Raton, FL, 2009).

[57] B.E. Sagan, The Symmetric Group: Representations, Combinatorial Algorithms, and Symmetric Functions (Springer Science \& Business Media, New York, 2013), Vol. 203.

[58] B. Koczor, Derangement Circuits, https://github.com/ QTechTheory/derangement_circuits, 2020 (refer to the webpage for an introduction: https:/qtechtheory.org/ derangement_circuits/).

[59] J. Dénes, The Representation of a Permutation as the Product of a Minimal Number of Transpositions and Its Connection with the Theory of Graphs, Publ. Math. Inst. Hung. Acad. Sci 4, 63 (1959).

[60] S. Bravyi, D. Gosset, R. Koenig, and M. Tomamichel, Quantum Advantage with Noisy Shallow Circuits, Nat. Phys. 16, 1040 (2020).

[61] S. Bravyi, D. Gosset, and R. König, Quantum Advantage with Shallow Circuits, Science 362, 308 (2018).

[62] M. Y. Niu, S. Lu, and I. L. Chuang, Optimizing QAOA: Success Probability and Runtime Dependence on Circuit Depth, arXiv:1905.12134.

[63] L. Zhou, S.-T. Wang, S. Choi, H. Pichler, and M. D. Lukin, Quantum Approximate Optimization Algorithm: Performance, Mechanism, and Implementation on Near-Term Devices, Phys. Rev. X 10, 021067 (2020).

[64] R. Babbush, N. Wiebe, J. McClean, J. McClain, H. Neven, and G. Kin-Lic Chan, Low-Depth Quantum Simulation of Materials, Phys. Rev. X 8, 011044 (2018).

[65] Z. Cai, Multi-Exponential Error Extrapolation and Combining Error Mitigation Techniques for NISQ Applications, arXiv:2007.01265.

[66] M. Silva, E. Magesan, D. W. Kribs, and J. Emerson, Scalable Protocol for Identification of Correctable Codes, Phys. Rev. A 78, 012347 (2008).

[67] E. Magesan, J. M. Gambetta, and J. Emerson, Characterizing Quantum Gates via Randomized Benchmarking, Phys. Rev. A 85, 042311 (2012).

[68] Z. Cai and S.C. Benjamin, Constructing Smaller Pauli Twirling Sets for Arbitrary Error Channels, Sci. Rep. 9, 11281 (2019). 
[69] Z. Cai, X. Xu, and S. C. Benjamin, Mitigating Coherent Noise Using Pauli Conjugation, npj Quantum Inf. 6, 17 (2020).

[70] F. B. Maciejewski, Z. Zimborás, and M. Oszmaniec, Mitigation of Readout Noise in Near-Term Quantum Devices by Classical Post-Processing Based on Detector Tomography, Quantum 4, 257 (2020).

[71] R. Nandkishore and D. A. Huse, Many-Body Localization and Thermalization in Quantum Statistical Mechanics, Annu. Rev. Condens. Matter Phys. 6, 15 (2015).

[72] D. J. Luitz, N. Laflorencie, and F. Alet, Many-Body Localization Edge in the Random-Field Heisenberg Chain, Phys. Rev. B 91, 081103(R) (2015).

[73] A. M. Childs, D. Maslov, Y. Nam, N. J. Ross, and Y. Su, Toward the First Quantum Simulation with Quantum Speedup, Proc. Natl. Acad. Sci. U.S.A. 115, 9456 (2018).

[74] G. Pagano, A. Bapat, P. Becker, K. S. Collins, A. De, P. W. Hess, H. B. Kaplan, A. Kyprianidis, W. L. Tan, C. Baldwin et al., Quantum Approximate Optimization of the LongRange Ising Model with a Trapped-Ion Quantum Simulator, Proc. Natl. Acad. Sci. U.S.A. 117, 25396 (2020).

[75] F. Arute, K. Arya, R. Babbush, D. Bacon, J. C. Bardin, R. Barends, S. Boixo, M. Broughton, B. B. Buckley, D. A. Buell et al., Quantum Approximate Optimization of NonPlanar Graph Problems on a Planar Superconducting Processor, Nat. Phys. 17, 332 (2021).

[76] D. Wecker, M. B. Hastings, and M. Troyer, Progress towards Practical Quantum Variational Algorithms, Phys. Rev. A 92, 042303 (2015).

[77] C. Cade, L. Mineh, A. Montanaro, and S. Stanisic, Strategies for Solving the Fermi-Hubbard Model on Near-Term Quantum Computers, Phys. Rev. B 102, 235122 (2020).

[78] R. Wiersema, C. Zhou, Y. de Sereville, J. F. Carrasquilla, Y. B. Kim, and H. Yuen, Exploring Entanglement and Optimization within the Hamiltonian Variational Ansatz, PRX Quantum 1, 020319 (2020).

[79] I. Pogorelov, T. Feldker, C. D. Marciniak, L. Postler, G. Jacob, O. Kriegelsteiner, V. Podlesnic, M. Meth, V. Negnevitsky, M. Stadler et al., A Compact Ion-Trap Quantum Computing Demonstrator, PRX Quantum 2, 020343 (2021).

[80] S. Khatri, R. LaRose, A. Poremba, L. Cincio, A. T. Sornborger, and P. J. Coles, Quantum-Assisted Quantum Compiling, Quantum 3, 140 (2019).

[81] H. F. Chau and F. Wilczek, Simple Realization of the Fredkin Gate Using a Series Of Two-Body Operators, Phys. Rev. Lett. 75, 748 (1995).

[82] J. A. Smolin and D. P. DiVincenzo, Five Two-Bit Quantum Gates Are Sufficient to Implement the Quantum Fredkin Gate, Phys. Rev. A 53, 2855 (1996).

[83] Z. Cai, Resource Estimation for Quantum Variational Simulations of the Hubbard Model, Phys. Rev. Applied 14, 014059 (2020).

[84] S. McArdle, S. Endo, A. Aspuru-Guzik, S. C. Benjamin, and X. Yuan, Quantum Computational Chemistry, Rev. Mod. Phys. 92, 015003 (2020).

[85] L. J. Stephenson, D. P. Nadlinger, B. C. Nichol, S. An, P. Drmota, T. G. Ballance, K. Thirumalai, J. F. Goodwin,
D. M. Lucas, and C. J. Ballance, High-Rate, High-Fidelity Entanglement of Qubits Across an Elementary Quantum Network, Phys. Rev. Lett. 124, 110501 (2020).

[86] X. Bonet-Monroig, R. Sagastizabal, M. Singh, and T. E. O'Brien, Low-Cost Error Mitigation by Symmetry Verification, Phys. Rev. A 98, 062339 (2018).

[87] P. Horodecki, From Limits of Quantum Operations to Multicopy Entanglement Witnesses and State-Spectrum Estimation, Phys. Rev. A 68, 052101 (2003).

[88] P. Horodecki, Measuring Quantum Entanglement without Prior State Reconstruction, Phys. Rev. Lett. 90, 167901 (2003).

[89] P. Horodecki and A. Ekert, Method for Direct Detection of Quantum Entanglement, Phys. Rev. Lett. 89, 127902 (2002).

[90] J. Fiurášek, M. Dušek, and R. Filip, Universal Measurement Apparatus Controlled by Quantum Software, Phys. Rev. Lett. 89, 190401 (2002).

[91] M. Dušek and V. Bužek, Quantum-Controlled Measurement Device for Quantum-State Discrimination, Phys. Rev. A 66, 022112 (2002).

[92] R. Filip, Overlap and Entanglement-Witness Measurements, Phys. Rev. A 65, 062320 (2002).

[93] U. Chabaud, E. Diamanti, D. Markham, E. Kashefi, and A. Joux, Optimal Quantum-Programmable Projective Measurement with Linear Optics, Phys. Rev. A 98, 062318 (2018).

[94] C. H. Bennett, G. Brassard, S. Popescu, B. Schumacher, J. A. Smolin, and W. K. Wootters, Purification of Noisy Entanglement and Faithful Teleportation via Noisy Channels, Phys. Rev. Lett. 76, 722 (1996).

[95] N. Kalb, A. A. Reiserer, P. C. Humphreys, J. J. Bakermans, S. J. Kamerling, N. H. Nickerson, S. C. Benjamin, D. J. Twitchen, M. Markham, and R. Hanson, Entanglement Distillation between Solid-State Quantum Network Nodes, Science 356, 928 (2017).

[96] D. Deutsch, A. Ekert, R. Jozsa, C. Macchiavello, S. Popescu, and A. Sanpera, Quantum Privacy Amplification and the Security of Quantum Cryptography Over Noisy Channels, Phys. Rev. Lett. 77, 2818 (1996).

[97] T. Jones and S. C. Benjamin, QuESTlink-Mathematica Embiggened by a Hardware-Optimised Quantum Emulator, Quantum Sci. Technol. 5, 034012 (2020).

[98] W. J. Huggins, S. McArdle, T. E. O’Brien, J. Lee, N. C. Rubin, S. Boixo, K. B. Whaley, R. Babbush, and J. R. McClean, Virtual Distillation for Quantum Error Mitigation, arXiv:2011.07064v1.

[99] Z. Cai, Quantum Error Mitigation Using Symmetry Expansion, arXiv:2101.03151.

[100] P. Czarnik, A. Arrasmith, L. Cincio, and P. J. Coles, Qubit-Efficient Exponential Suppression of Errors, arXiv: 2102.06056.

[101] T. D. Nguyen and R. Van Meter, A Space-Efficient Design for Reversible Floating Point Adder in Quantum Computing, arXiv:1306.3760.

[102] B. Foxen et al. (Google AI Quantum), Demonstrating a Continuous Set of Two-Qubit Gates for Near-Term Quantum Algorithms, Phys. Rev. Lett. 125, 120504 (2020). 
[103] A. Fedorov, L. Steffen, M. Baur, M. P. da Silva, and A. Wallraff, Implementation of a Toffoli Gate with Superconducting Circuits, Nature (London) 481, 170 (2012).
[104] H. R. Grimsley, S. E. Economou, E. Barnes, and N. J. Mayhall, An Adaptive Variational Algorithm for Exact Molecular Simulations on a Quantum Computer, Nat. Commun. 10, 3007 (2019). 